\title{
Sex-specific tuning of modular muscle activation patterns for locomotion in
}

\section{young and older adults}

\section{Authors}

5 Alessandro Santuz ${ }^{1,2^{*}}$, Lars Janshen ${ }^{1,2}$, Leon Brüll ${ }^{1,2,3}$, Victor Munoz-Martel ${ }^{1,2}$, Juri Taborri ${ }^{4}$, Stefano Rossi ${ }^{4}$, and Adamantios Arampatzis ${ }^{1,2}$

\section{Affiliations}

${ }^{1}$ Department of Training and Movement Sciences, Humboldt-Universität zu Berlin, 10115 Berlin,

10 Germany

${ }^{2}$ Berlin School of Movement Science, Humboldt-Universität zu Berlin, 10115 Berlin, Germany

${ }^{3}$ Network Aging Research, Heidelberg University, 69117 Heidelberg, Germany

${ }^{4}$ Department of Economics, Engineering, Society and Business Organization (DEIM), University of Tuscia, 01100 Viterbo, Italy

\section{Contact Info}

*Correspondence: alessandro.santuz@hu-berlin.de

Twitter handle: @alesantuz 


\section{Abstract}

There is increasing evidence that including sex as a biological variable is of crucial importance to promote rigorous, repeatable and reproducible science. In spite of this, the body of literature that accounts for the sex of participants in human locomotion studies is small and often produces controversial results. Here, we investigated the modular organization of muscle activation patterns

25 for human locomotion using the concept of muscle synergies with a double purpose: i) uncover possible sex-specific characteristics of motor control and ii) assess whether these are maintained in older age. We recorded electromyographic activities from 13 ipsilateral muscles of the lower limb in young and older adults of both sexes walking (young and old) and running (young) on a treadmill. The data set obtained from the 215 participants was elaborated through non-negative matrix factorization to extract the time-independent (i.e., motor modules) and time-dependent (i.e., motor primitives) coefficients of muscle synergies. We found sparse sex-specific modulations of motor control. Motor modules showed a different contribution of hip extensors, knee extensors and foot dorsiflexors in various synergies. Motor primitives were wider (i.e., lasted longer) in males in the propulsion synergy for walking (but only in young and not in older adults) and in the

35 weight acceptance synergy for running. Moreover, the complexity of motor primitives was similar in younger adults of both sexes, but lower in older females as compared to older males. In essence, our results revealed the existence of small but defined sex-specific differences in the way humans control locomotion and that these strategies are not entirely maintained in older age. 


\section{Introduction}

For many decades, the vast majority of basic and clinical research studies using non-human animals failed to either include females or to consider sex as a biological variable, often without scientifically funded reason [1-3]. In the last few years, and especially in human research, this

45 trend started changing [2]. Scientists are becoming more and more aware that the physiological differences between males and females must be accounted for in order to promote rigorous, repeatable and reproducible science [4,5].

Human locomotion, with its highly stereotyped patterns, has been an ideal subject of neuroscience and biomechanics studies for more than one century [6]. Some sex-specific characteristics, often

50 too subtle to quantify [7,8], of human walking and running have been unraveled, such as the different hip-, knee- and ankle-joint kinematics or the body height-dependent preferred speed and step frequency [9-11]. Females have been shown to walk with greater hip flexion and lower knee extension at touchdown than males, generating greater mechanical joint power from those two joints during the propulsion phase [9]. Moreover, older females showed smaller range of motion

55 of the hip joint during walking in the sagittal plane, the opposite being true in the frontal plane [10]. In younger participants these findings were reversed [7] and a greater arm swing and pelvic obliquity in the frontal plane was noted in females. In general, it is clear that the quantitative assessment of anecdotal and qualitative observations is difficult to obtain and only few studies endeavored to rigorously quantify the sex specificity of kinematic and kinetic parameters during

60 locomotion [8]. Similarly, only a few studies analyzed the way in which females and males activate muscles to produce locomotion. Some of those works reported no effect of sex on the way muscles are activated during either walking [12-17] or running $[18,19]$. Others reported higher or earlier muscle activations in females than in males during either walking [19-22] or running [23-26], 
with females sometimes showing a higher coactivation of agonist and antagonists muscles during

65 walking $[27,28]$. However, all the mentioned studies only considered a limited amount of muscles (from a minimum of one and until a rare maximum of eight), thus limiting further analysis of the coordination between muscle groups during the various phases of the gait cycles. Moreover, it might be relevant to understand if potential sex-specific muscle coordination patterns are maintained in older age, since it is known that the neuromotor strategies used by young people differ from those employed by older adults [16,22,29-34].

The purpose of this study was to investigate the muscle activation patterns for locomotion in males and females of different ages in order to: i) find possible sex-specific characteristics of motor control and ii) assess whether sex similarities or differences in movement coordination are maintained in older age. To investigate the first point, we adopted the framework of muscle

75 synergies. Based on the hypothesis that the central nervous system can simplify the production of movement by activating muscles in coordinated groups rather than individually [35], muscle synergies [36] are extracted from electromyographic (EMG) activities. In the past twenty years, a number of scientific works adopted the concept of muscle synergies and their modular organization to better understand the neurophysiological mechanisms underlying the generation of locomotor

80 patterns. From the basic comparison of different locomotion modes [37] to the more elaborate investigations on development [38], aging [33] and pathology [39], muscle synergies are a widespread tool for the assessment of motor coordination during walking and running. Yet, to the best of our knowledge, sex as a biological variable has never been the focus of a scientific work analyzing muscle synergies for locomotion. We recorded the EMG activity of 13 lower limb 85 muscles (right side) in females and males during locomotion and then extracted the timeindependent (i.e., motor modules) and time-dependent (i.e., motor primitives) coefficients of 
muscle synergies through non-negative matrix factorization. To assess the possible influence of aging on sex-specific coordination patterns, we analyzed walking in young and older adults as well as running in young adults. We hypothesized that small, if any, sex-specific modulations would

90 emerge from the analysis of muscle synergies and of gait temporal parameters. Moreover, based on previous findings [30,31,33], we hypothesized that any sex-related tuning of muscle synergies might depend on the age group of the participants. 


\section{Results}

\section{Gait cycle temporal parameters}

The duration of the stance phase (Figure 1 and Figure 2A) during walking was moderately affected by both sex $(\beta=-35.57, \operatorname{CrI}=[-58.65,-12.33])$ and age $(\beta=15.95, \operatorname{CrI}=[-1.04,32.79])$, with an interaction between the two. The post-hoc analysis revealed a stronger effect of sex on the stance duration in older adults, while in both age groups females spent less time than males in the stance

100 phase (Figure S1). It might look like the different walking speeds played an important role (Figure 2A). However, this effect was associated with a large estimation uncertainty, as visible from the large width of the credible interval $(\beta=160.86, \mathrm{CrI}=[52.90,269.44])$. Specifically, the walking speed in young adults ranged between 1.1 and $1.5 \mathrm{~m} / \mathrm{s}$, while in older adults it spanned between 1.0 and $1.4 \mathrm{~m} / \mathrm{s}$ (see also Table 1 ). In running, sex had a negligible effect on the stance times $(\beta=$ 105 1.48, $\mathrm{CrI}=[-15.22,18.76])$ and the different locomotion speed was also of little importance $(\beta=$ -2.95, CrI $=[-30.31,25.03])$. Running speeds ranged between 2.0 and $3.8 \mathrm{~m} / \mathrm{s}$ (see also Table 1 ). The swing duration (Figure 1 and Figure 2B) was moderately affected by both sex $(\beta=-16.8$, CrI $=[-34.01,0.34])$ and age $(\beta=-15.66, \operatorname{CrI}=[-28.22,-3-31])$, with negligible interaction between the two. Regardless of age, females showed shorter swing duration than males, while older adults

110 had shorter swing times than younger participants. In running, swing duration was not clearly affected by sex $(\beta=5.41, \operatorname{CrI}=[-13.10,25.19])$, but the locomotion speed had a moderate effect on this parameter $(\beta=31.88, \mathrm{CrI}=[1.25,64.25])$.

For cadence, or number of steps per minute (Figure 1 and Figure 2C), there was only a small (if any) effect of sex $(\beta=0.44 \operatorname{CrI}=[-2.98,3.92])$ and age $(\beta=-1.98, \operatorname{CrI}=[-4.52,0.61])$ in walking

115 with negligible interaction and a moderate effect of sex $(\beta=4.03, \operatorname{CrI}=[-0.32,8.47])$ and speed $(\beta=4.65, \mathrm{CrI}=[-2.59,12.06])$ in running. The considerations about the effect of walking speed 
are also valid for the swing times $(\beta=62.94, \mathrm{CrI}=[-14.78,142.34])$ and cadence $(\beta=13.21, \mathrm{CrI}$ $=[-3.30,29.41]$, Figure 2B and C).

In summary, the effects of sex and age on stance duration, swing duration and cadence were uncertainly estimated) role in walking than in running.

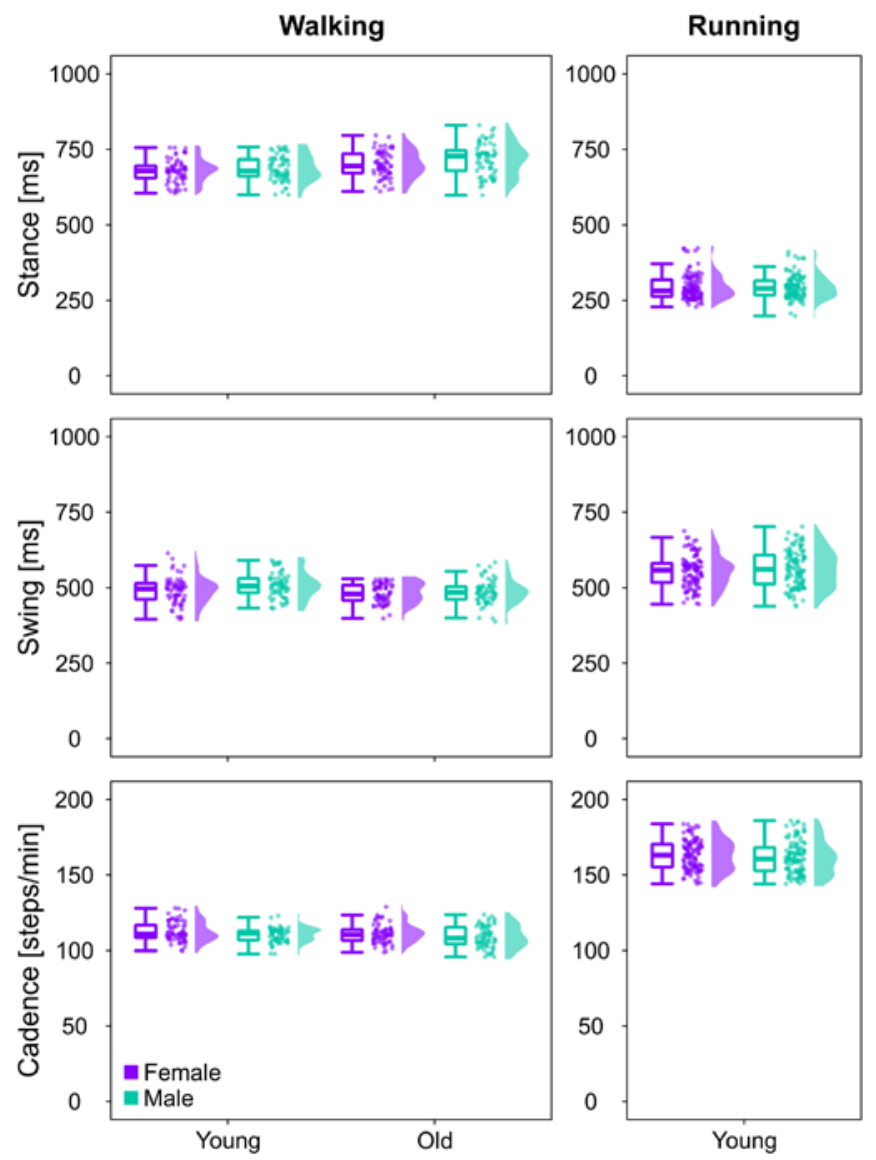

Figure 1. Gait temporal parameters. Boxplots describing the three gait temporal parameters stance duration, swing duration and cadence for the three investigated groups, calculated from walking or running. Raw data points (dots represent the mean value of 30 gait cycles from every participant) and their density estimates are presented to the right side of each boxplot. 

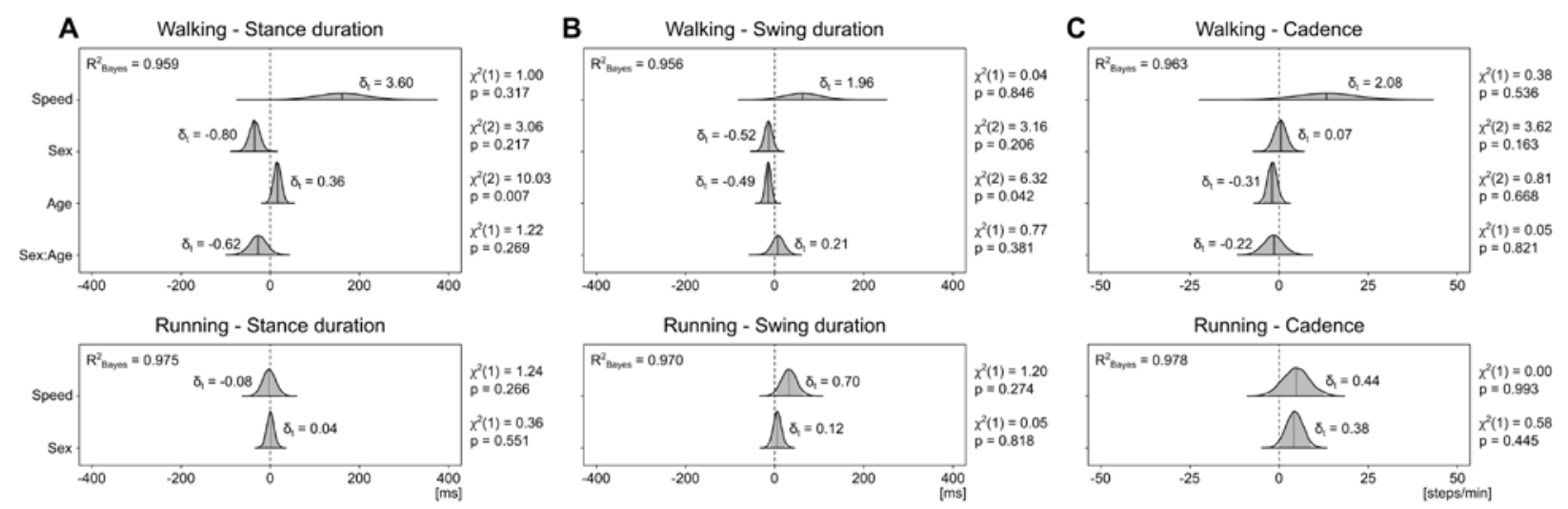

Figure 2. Posterior $95 \%$ credible intervals for the estimated differences in gait temporal

parameters. (A) The 95\% credible intervals and their probability distributions (shaded areas)

130 describe the effects and interaction of sex and age (with locomotion speed as a covariate) on the stance duration. Positive values indicate longer stance times in females than in males and in older than in younger adults. Effect size in the style of Hedges are shown as $\delta_{\mathrm{t}}$. A Bayesian equivalent of $\mathrm{R}^{2}$ is presented as $\mathrm{R}^{2}$ Bayes. The $\chi^{2}$ and $\mathrm{p}$-values resulting from a frequentist linear mixed model analysis are reported as additional information. (B) The same as in panel (A), but for the swing 135 phase duration. (C) The same as in panel (A), but for the cadence or number of steps per minute.

\section{$\underline{\text { Muscle synergies }}$}

The activity of 13 muscles of the lower limb (Figure 3) could be factorized into four task-related muscle synergies in all groups (Figure 4) with low to negligible effects of sex (walking: $\beta=0.00$,

$\mathrm{CrI}=[-0.02,0.02], \delta_{\mathrm{t}}=0.01$; running: $\left.\beta=0.01, \mathrm{CrI}=[0.00,0.02], \delta_{\mathrm{t}}=0.33\right)$ or age $(\beta=-0.01$,

$\left.\mathrm{CrI}=[-0.02,0.00], \delta_{\mathrm{t}}=-0.25\right)$ on the reconstruction quality $\left(\mathrm{R}^{2}\right.$ walking, young females $=0.83 \pm 0.03$;

$\mathrm{R}^{2}$ walking, young males $=0.82 \pm 0.03 ; \mathrm{R}^{2}$ walking, old females $=0.83 \pm 0.03 ; \mathrm{R}^{2}$ walking, old males $=0.82 \pm 0.03$;

$\mathrm{R}_{\text {running, young females }}^{2}=0.85 \pm 0.03 ; \mathrm{R}^{2}$ running, young males $=0.85 \pm 0.03$ ). The first muscle synergy described the acceptance of the body weight, with a major involvement of the knee extensors and 
145 hip muscles. The second synergy was functionally related to the propulsion phase, mainly managed by the plantarflexors (i.e., the extensors of the ankle joint). The third synergy represented the early stage of the swing phase, mostly orchestrated by the dorsiflexors (i.e., the ankle flexors). The fourth and chronologically last synergy was that related to the late swing phase, when mostly the knee flexors were contributing.

150 By looking at the motor modules (Figure 5, Figure S2), it is possible to identify the different relative contribution of muscles to a specific synergy. In walking, females showed a bigger contribution of the gluteus maximus than males in all synergies except for the late swing, where the contribution was similar. This was not the case in running, where the hip extensor was contributing similarly in both sexes in all synergies except for the late swing, were the contribution

155 in females was lower than in males. The gluteus medius was more used by males during the weight acceptance of walking, while the opposite happened during the early swing in running. The tensor fascice latce was similarly employed by both sexes during running, but in walking females showed a lower contribution of this muscle. The knee extensors behaved largely similar, but in both walking and running females used less vastus medialis during the weight acceptance and less 160 rectus femoris during the early swing. The knee flexors were more important for the late swing phase of walking in females than in males, behaving generally similar in all the other synergies. Females used less tibialis anterior in the late swing synergy in walking, but used more of it during the same synergy in running. Finally, the plantarflexors were similarly managed by females and males, at least in the propulsion synergy that saw them mostly involved.

165 The coactivation between flexor and extensor muscles around each of the three considered joints (i.e., hip, knee and ankle, see Figure 5), revealed sparse effects of sex in both walking and running. In walking, a moderate effect of sex on coactivation was visible in the propulsion synergy, with 
females showing less coactivation than males $\left(\beta=-0.08, \operatorname{CrI}=[-0.13,-0.04], \delta_{t}=-0.37\right)$. However, the post-hoc analysis (Figure S3) revealed that this difference was mostly due to the coactivation

170 of muscles acting around the hip and the knee, two joints that are not as relevant as the ankle for the propulsion phase. Conversely, in running females showed moderately higher coactivation than males in the early swing $\left(\beta=0.1, \mathrm{CrI}=[0.03,0.17], \delta_{\mathrm{t}}=0.33\right)$ and late swing $(\beta=0.1, \mathrm{CrI}=[0.05$, $\left.0.15], \delta_{\mathrm{t}}=0.34\right)$ synergies. While in the early swing the effect was due to the muscles acting around the joint important for that synergy (i.e., the ankle), in the late swing the differences were due 175 mainly to the hip and ankle, two joints that were less involved than the knee in the task of stopping the leg before touchdown (Figure S3). The effect of ageing on coactivation had small to none interaction with sex and was of moderate effect in three out of four synergies: the propulsion $(\beta=$ $\left.0.06, \mathrm{CrI}=[0.02,0.1], \delta_{\mathrm{t}}=0.25\right)$, the early swing $\left(\beta=0.07, \mathrm{CrI}=[0.03,0.1], \delta_{\mathrm{t}}=0.36\right)$ and the late swing $\left(\beta=-0.14, \mathrm{CrI}=[-0.18,-0.1], \delta_{\mathrm{t}}=-0.58\right)$.

180 Concerning the motor primitives, the most important effect on the width (i.e., full width at half maximum, Figure 6) and timing of the main activation (i.e., center of activity, Figure 6) during walking was the age of the participants (Figure 7). Specifically, aging had a large effect on increasing the width of all four primitives (weight acceptance: $\beta=7.51$, CrI $=[5.29,9.74]$; propulsion: $\beta=6.53, \mathrm{CrI}=[3.36,9.70]$; early swing: $\beta=12.75, \mathrm{CrI}=[8.34,17.13]$; late swing: $\beta$ $185=12.39, \mathrm{CrI}=[9.26,15.46])$. Moreover, ageing had a moderate to large effect on shifting earlier the main activity's timing of all motor primitives (propulsion: $\beta=-7.24, \mathrm{CrI}=[-9.51,-5.02]$; early swing: $\beta=-15.48, \operatorname{CrI}=[-23.78,-7.01]$; late swing: $\beta=-17.14, \operatorname{CrI}=[-27.42,-6.6])$ except the weight acceptance $(\beta=5.32, \mathrm{CrI}=[-3.44,14.3])$. However, the sex of the participants did not result in such large changes (Figure 7). Females showed a moderately narrower (i.e., shorter) 190 propulsion primitive $(\beta=-3.06, \mathrm{CrI}=[-6.98,0.81])$. A post-hoc investigation of the yet weak 
interaction of sex and age showed that the narrowing in females mostly happened in young participants and not in older ones (Figure S4). Moreover, sex had a moderate effect on the timing of the early swing primitive, which in females was located earlier in time than in males $(\beta=-10.61$, $\mathrm{CrI}=[-21.32,0.06])$. In running, sex had a small effect on the width of the weight acceptance 195 primitive, which was reduced in females $(\beta=-1.49$, $\mathrm{CrI}=[-3.66,0.69])$. All the other effects were smaller and the same considerations done above for the effect of the different locomotion speeds are valid also for the full width at half maximum and center of activity of motor primitives.

In summary, the analysis of motor modules revealed largely similar relative muscle contributions in females and males, but with some sparse differences involving mostly the hip extensors, knee extensors and foot dorsiflexors. Likewise, we found sparse sex-related differences on the coactivation of synergistic muscles, while motor primitives resulted wider in males in the propulsion synergy for walking (but only in young and not in older adults) and in the weight acceptance synergy for running. 


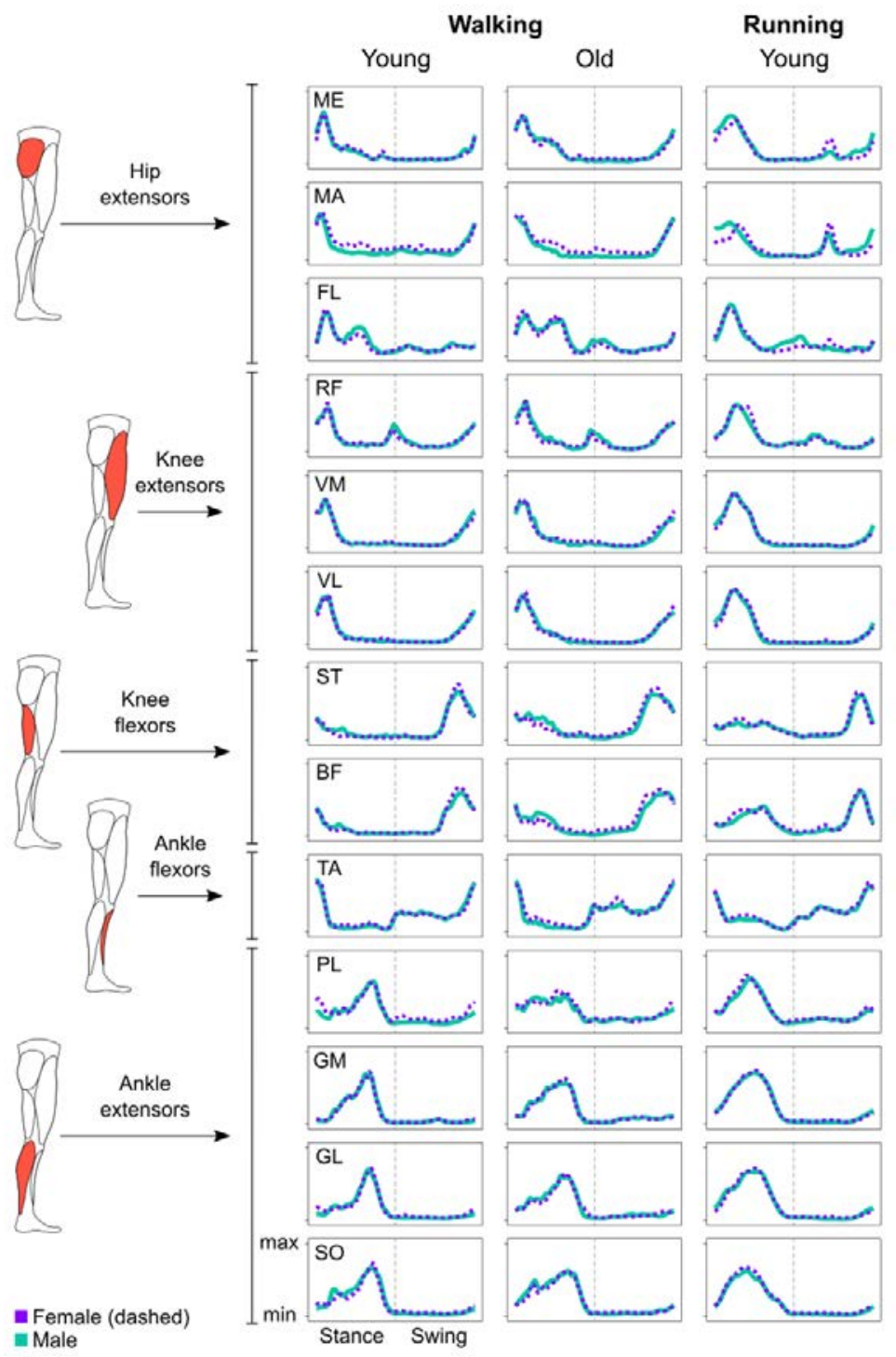

Figure 3. Average muscle activity. The mean activations of the recorded muscles are presented for the stance and swing phases of walking (young and old adults) and running (young adults) for female and male participants. Signals were rectified, filtered, time- and amplitude normalized as described in the methods. Muscle abbreviations: $\mathrm{ME}=$ gluteus medius, $\mathrm{MA}=$ gluteus maximus, $\mathrm{FL}=$ tensor fasciæ latæ, $\mathrm{RF}=$ rectus femoris, $\mathrm{VM}=$ vastus medialis, $\mathrm{VL}=$ vastus lateralis, $\mathrm{ST}=$ semitendinosus, $\mathrm{BF}=$ biceps femoris, $\mathrm{TA}=$ tibialis anterior, $\mathrm{PL}=$ peroneus longus, $\mathrm{GM}=$ gastrocnemius medialis, GL = gastrocnemius lateralis, $\mathrm{SO}=$ soleus. 


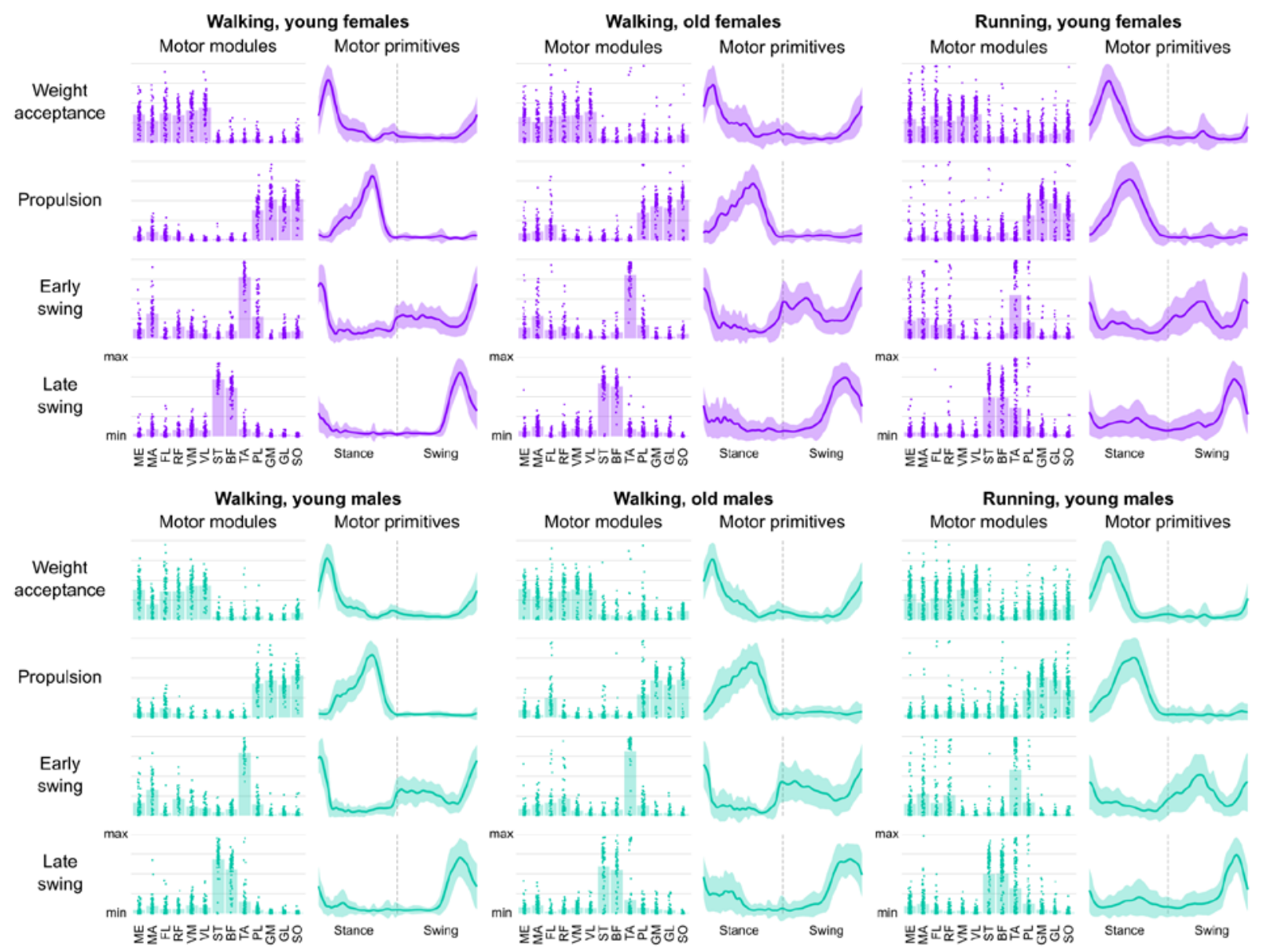

Figure 4. Muscle synergies. The motor modules are presented on a normalized y-axis base: each muscle contribution within one synergy can range from 0 to 1 and each dot represents individual

215 trials. For the mean motor primitives (shaded standard deviation), the x-axis full scale represents the averaged gait cycle (with stance and swing normalized to the same amount of points and divided by a vertical line) and the $\mathrm{y}$-axis the normalized amplitude. Muscle abbreviations: $\mathrm{ME}=$ gluteus medius, $\mathrm{MA}=$ gluteus maximus, $\mathrm{FL}=$ tensor fasciæ latæ, $\mathrm{RF}=$ rectus femoris, $\mathrm{VM}=$ vastus medialis, $\mathrm{VL}=$ vastus lateralis, $\mathrm{ST}=$ semitendinosus, $\mathrm{BF}=$ biceps femoris, $\mathrm{TA}=$ tibialis 220 anterior, $\mathrm{PL}=$ peroneus longus, $\mathrm{GM}$ = gastrocnemius medialis, $\mathrm{GL}=$ gastrocnemius lateralis, $\mathrm{SO}$ = soleus. 


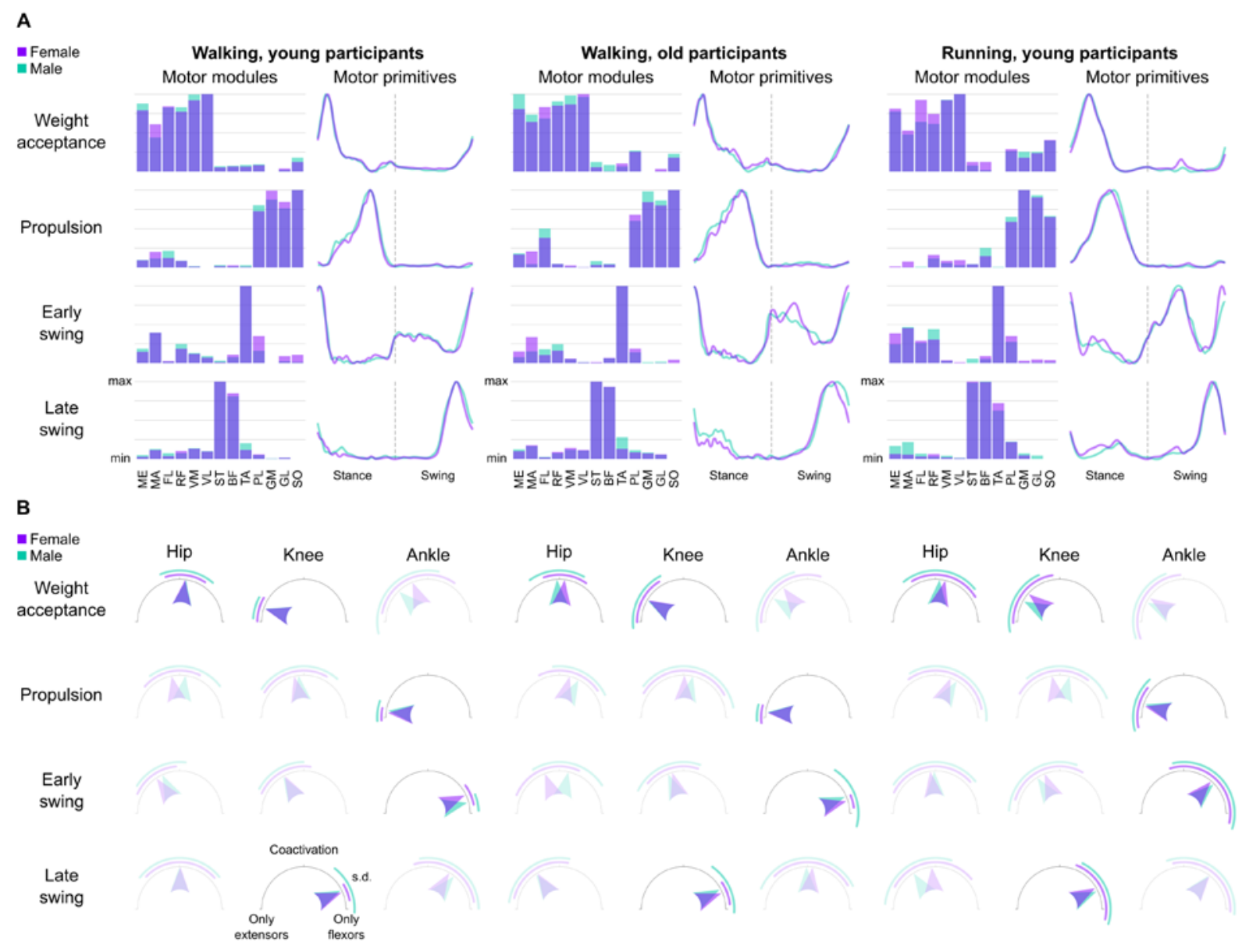

Figure 5. Overlayed muscle synergies and coactivation index. (A) The average muscle synergies of females and males are overlayed for comparison. See caption of Figure 4 for details.

225 Muscle abbreviations: Muscle abbreviations: $\mathrm{ME}=$ gluteus medius, $\mathrm{MA}=$ gluteus maximus, $\mathrm{FL}=$ tensor fasciæ latæ, $\mathrm{RF}=$ rectus femoris, $\mathrm{VM}=$ vastus medialis, $\mathrm{VL}=$ vastus lateralis, $\mathrm{ST}=$ semitendinosus, $\mathrm{BF}=$ biceps femoris, $\mathrm{TA}=$ tibialis anterior, $\mathrm{PL}=$ peroneus longus, $\mathrm{GM}=$ gastrocnemius medialis, $\mathrm{GL}=$ gastrocnemius lateralis, $\mathrm{SO}=$ soleus. $(\mathrm{B})$ Coactivation index calculated from the motor modules. Joints crossed by the most important muscles contributing to 230 each muscle synergy are highlighted. Arrows pointing to the left show that muscle activity comes exclusively from extensors, while arrows pointing to the right mean that activity comes only from 
flexors. Arrows pointing upwards indicate perfect coactivation of flexors and extensors, meaning that the average module values for the flexors of that joint are the same as those for the extensors.

A
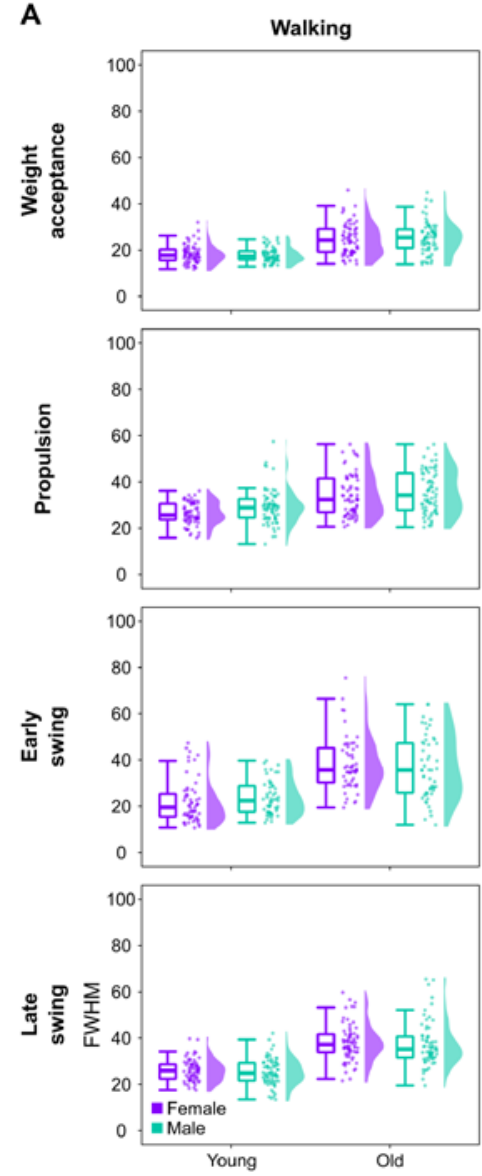
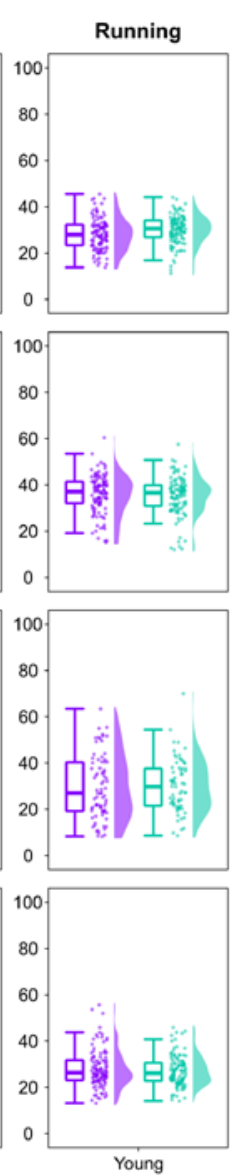

B
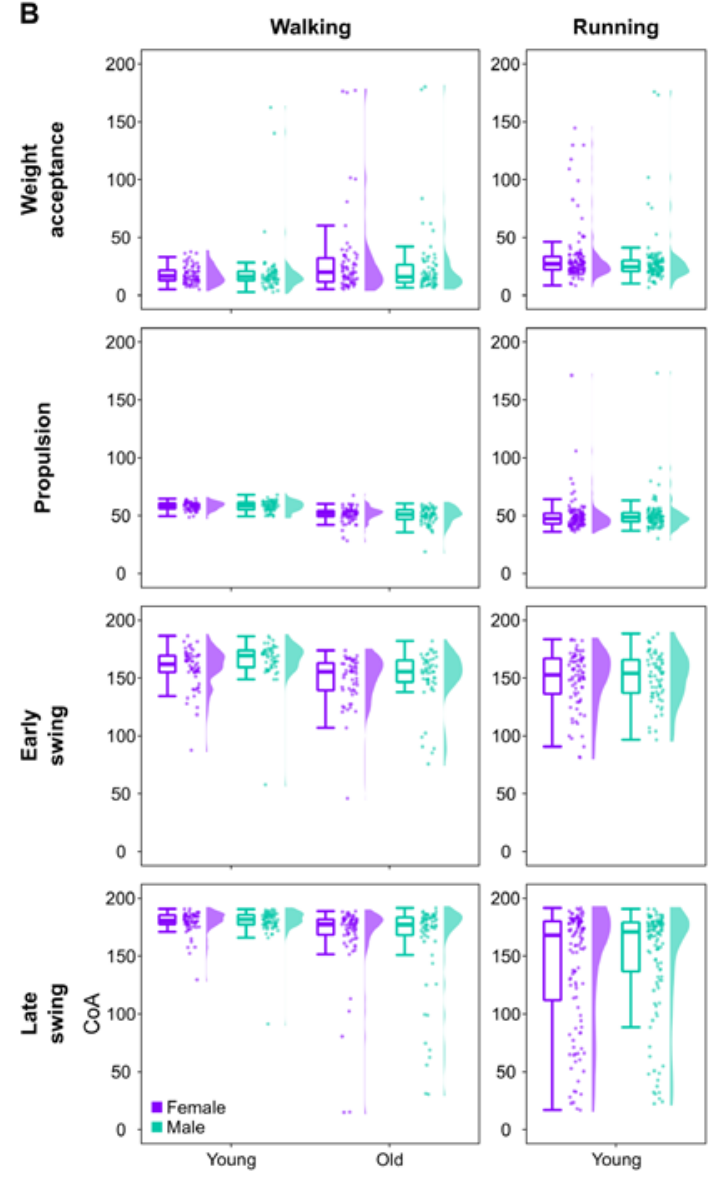

Figure 6. Full width at half maximum and center of activity of motor primitives. (A) Boxplots

describing the full width at half maximum (FWHM) of the extracted motor primitives. Raw data points (dots represent the mean value of 30 gait cycles from every participant) and their density estimates are presented to the right side of each boxplot. (B) The same as in panel (A), but for the center of activity (CoA). 

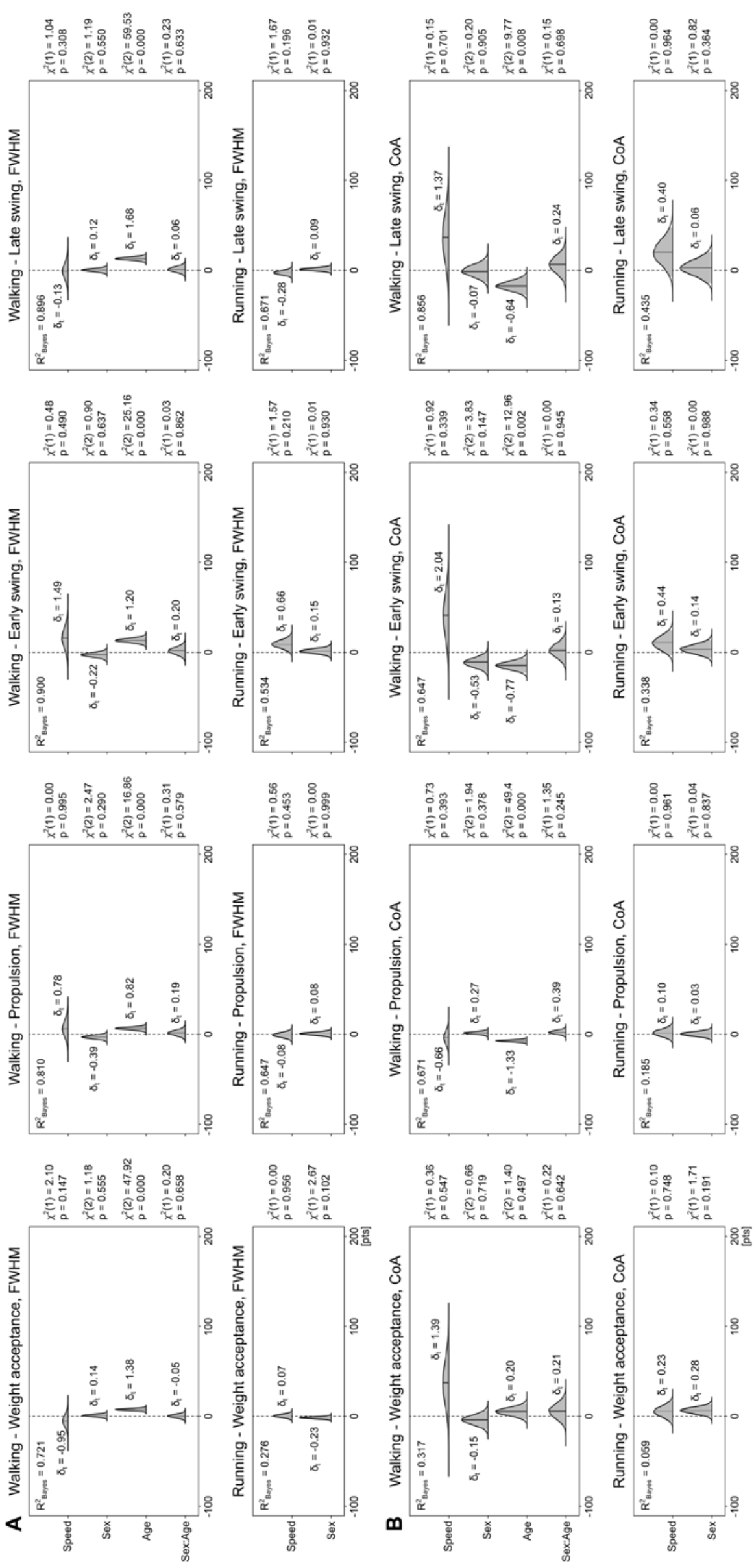
Figure 7. Posterior $95 \%$ credible intervals for the estimated differences in full width at half

maximum and center of activity. (A) The 95\% credible intervals and their probability distributions (shaded areas) describe the effects and interaction of sex and age (with locomotion speed as a covariate) on the full width at half maximum (FWHM). Positive values indicate wider

245 primitives in females than in males and in older than in younger adults. Effect size in the style of Hedges (i.e., considering all the variance sources in the model) are shown on the graphs and called $\delta_{\text {t }}$ A Bayesian equivalent of $\mathrm{R}^{2}$ (here $\mathrm{R}^{2}$ Bayes) describes how well the model fits the data. Next to each estimation, the $\chi^{2}$ and $p$-values resulting from a frequentist linear mixed model analysis are reported as additional information. (B) The same as in panel (A), but for the center of activity 250 (CoA).

\section{Muscle synergy dynamics}

The fractal analysis of motor primitives (Figure 8) did not reveal any large effect of either age or sex on the local (i.e., Higuchi's fractal dimension) and global (i.e., Hurst exponent) complexity

255 (Figure 9). In walking, sex had a small effect on both the local and global complexity, reducing them in females (local: $\beta=-0.01, \operatorname{CrI}=[-0.04,0.01]$; global: $\beta=-0.01$, $\operatorname{CrI}=[-0.05,0.02]$ ). However, we could observe a moderate interaction between sex and age (local: $\beta=-0.04, \mathrm{CrI}=[-$ 0.08, 0.00]; global: $\beta=-0.04, \mathrm{CrI}=[-0.08,0.01])$, the post-hoc analysis of which revealed that older females tended to reduce more than the younger counterparts both the local and global 260 complexity as compared to males (Figure S5).

In summary, young adults did not show sex-related differences in the local and global complexity of motor primitives, but this changed in older adults indicating that aging might influence the way females and males modulate the dynamics of motor primitives. 
A
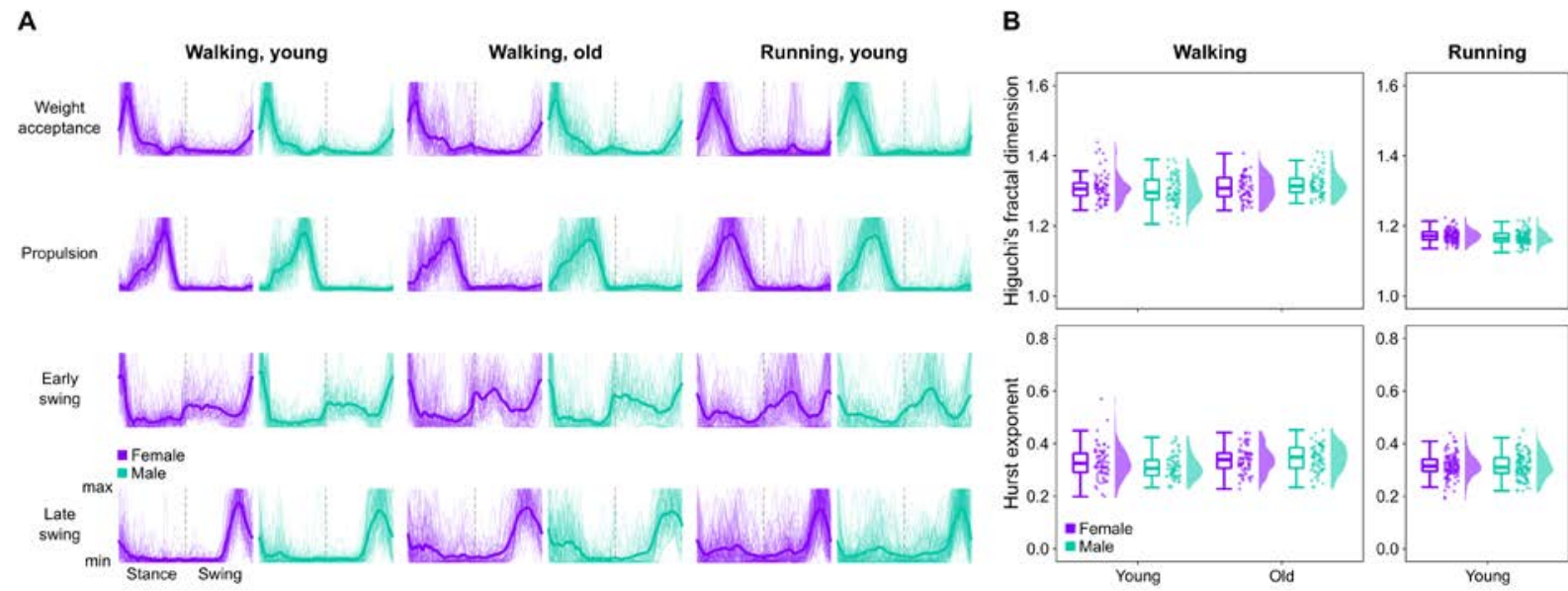

Figure 8. Motor primitives and their fractal properties. (A) Overall average motor primitives (thick lines) and each participant’s average motor primitive (thin lines). (B) Boxplots describing the Higuchi's fractal dimension and the Hurst exponent of the extracted motor primitives. Raw data points (dots represent the mean value from every participant) and their density estimates are presented to the right side of each boxplot.
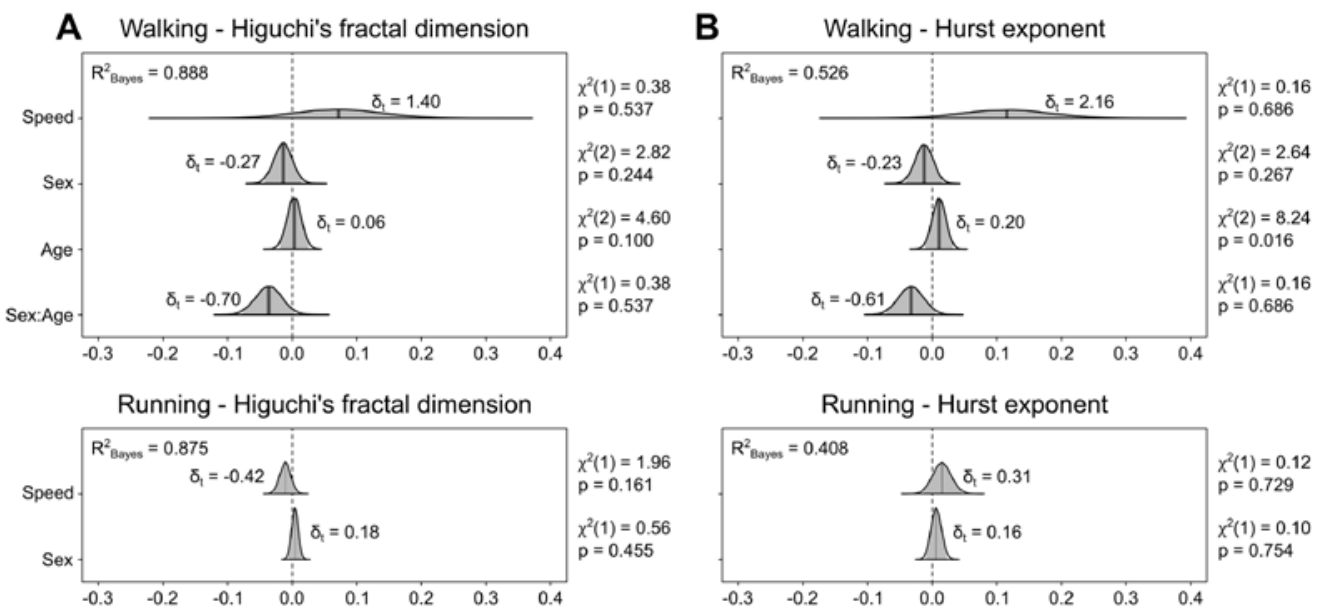

Figure 9. Posterior 95\% credible intervals for the estimated differences in Higuchi's fractal dimension and Hurst exponent. (A) The 95\% credible intervals and their probability distributions (shaded areas) describe the effects and interaction of sex and age (with locomotion speed as a covariate) on the Higuchi's fractal dimension. Positive values indicate higher fractal dimension in 
275 females than in males and in older than in younger adults. Effect size in the style of Hedges (i.e., considering all the variance sources in the model) are shown on the graphs and called $\delta_{\mathrm{t}}$. A Bayesian equivalent of $\mathrm{R}^{2}$ (here $\mathrm{R}^{2}$ Bayes) describes how well the model fits the data. Next to each estimation, the $\chi^{2}$ and $\mathrm{p}$-values resulting from a frequentist linear mixed model analysis are reported as additional information. (B) The same as in panel (A), but for the Hurst exponent. 


\section{Discussion}

The investigation of sex as a biological variable is of crucial importance for the equitable assessment of human performance, pathology, aging effects, and so forth. Here, we investigated the effects of sex - and how these may be influenced by aging - on the modular organization of

285 muscle activity during locomotion. We hypothesized and subsequently found that, in general, there is high similarity between sexes in gait timing and synergistic muscle activity. The sparse but potentially important sex-specific modulations of motor control found in this study might be leveraged in the future to deepen the neurophysiological mechanisms that regulate sex-related diversity in the response to exercise.

290 When walking on a treadmill at a speed close to the average preferred one, females showed shorter swing times than males, regardless of age. Older adults showed shorter swing and longer stance times than younger participants, thus the overall cadence was not affected. In running, where we only analyzed younger adults, sex did not play a big role on the stance and swing times, while cadence was moderately higher in females than in males. This first part of the results shows that,

295 in the considered participants, sex-related differences were limited to the swing times in walking and cadence in running and they did not vary with aging, largely in agreement with previous literature $[11,13,22,40]$.

The four synergies that described the phases of weight acceptance, propulsion, early swing and late swing were found independently of sex, age group or locomotion types, in accordance with most of the available body of existing literature that, however, does not include any study that specifically investigated sex as a biological variable during locomotion [31,33,37,41,42]. Some previous works that analyzed non-factorized EMG activities from the lower limbs found that females and males shared similar muscle activations during both walking [12-17] and running 
$[18,19]$. However, subtler sex-related differences were found in other works. For instance, females have been known to show higher hip extensor, knee extensor, foot dorsiflexor or foot plantarflexor activity than males during walking [20-22] or running [23-25]. Although motor modules, due to data normalization, describe the relative contribution of one muscle with respect to the others in a certain synergy and not the absolute amplitude of the signal, our analysis could partially confirm those previous findings. The identified sex-specific contributions of the hip, knee and ankle joint 310 muscles to the relevant synergies, even though sparse, could potentially be useful for the design of locomotion-based experimental protocols or training interventions that account for the biological sex of the participants. On a related note, it has been previously reported that females might show more preactivation than males in muscles such as the knee flexor biceps femoris in walking [19] and the foot evertor and plantarflexor peroneus longus in running [26]. The two muscles are 315 respectively contributing to the propulsion synergy for walking and late swing synergy for running. Preactivation is a time-related strategy, defined as an activation happening before the functional use of the muscle. However, our analysis of the width and main peak timing revealed that the propulsion primitive for walking and the weight acceptance primitive for running were narrower, but not time-shifted, in females than in males. Thus, our results do not support a preactivation of 320 the knee flexors (mainly important for the late swing synergy) in walking and of foot plantarflexors (mainly important for the propulsion synergy) in running, at least at a muscle-group level. A narrowing without modification of the main peak timing is yet an interesting outcome that implies shorter - and not time-shifted - basic activation patterns in females than in males in two synergies that occur during stance. This finding is additionally interesting given the high similarity of the 325 stance duration between the two sexes, implying that the narrowing happened in absolute terms (i.e., given the same stance duration, females spent less time than males in the propulsion phase of 
walking and weight acceptance phase of running). An explanation of this phenomenon cannot be directly supported by the results of this study, but it is possible to speculate on its potential neurophysiological importance based on present and past results.

330 The temporal widening of motor primitives, but also of muscle-specific activation patterns, has been previously interpreted as a compensatory mechanism adopted by the central nervous system to cope with the challenges imposed by: a) internal perturbations deriving from pathology [43-45] or sensory feedback impairment [46], b) external mechanical perturbations [31,47-49], c) high locomotion speeds [50], d) development stage [38,45] or e) aging [30,31], the latter being

335 confirmed by the results of the present study. Here, we recruited healthy, young adults of both sexes and had them walk or run at similar submaximal speeds. It is thus unlikely that the sexspecific width of the stance phase primitives was linked to any of the aforementioned factors. Our results show that muscles were being activated for a shorter amount of time in females than in males during the stance phase of locomotion, when muscle activities are at their highest (and so

340 are energy requirements). Yet, the shorter activation does not translate in lower energy consumption since it is known that, in elite athletes running at similar submaximal speeds, males are more economical than females in terms of absolute oxygen uptake and no sex specificity exists when the oxygen uptake is normalized by the body mass [51]. Moreover, despite previous sporadic findings of higher muscle coactivation in females than in males during walking $[27,28]$, here we

345 showed that females had lower coactivation at the ankle joint during the propulsion phase of walking, while during running the weight acceptance showed similar coactivation levels. However, females are known to possess superior resistance to fatigue after prolonged whole-body submaximal exercise [4,52-55], thus the narrowing of motor primitives (i.e., reduced utilization 
over time) associated with the demanding stance phase of locomotion might constitute a neuromotor strategy to limit fatigability during prolonged locomotion efforts.

With our analysis, we could also show that sex specificities were generally maintained through aging, but not in all parameters. For instance, the shorter stance phase in males than in females was more evident in participants of older age than in the younger ones. Conversely, the narrower propulsion primitive for walking in females was more evident in younger than in older participants.

355 Last but not least, both the local and global complexity of motor primitives were lower in older females than in older males, but this would not hold for the younger participants. These outcomes indicate that sex-specific strategies are not be necessarily retained through aging, pointing to the possibility that the course of motor optimization over lifespan is sex-dependent and needs to be considered when designing therapy or exercise protocols for quality-of-life improvement.

360 In conclusion, the albeit subtle sex-specific modulations of motor control found in this study justify on several levels the need to continue designing research protocols based on mixed cohorts [5]. Physiological discrepancies between males and females are known to affect the response to acute and long-term exercise and it is likely that interventions designed based on data coming from male participants might not fit the physiology of both sexes [4]. This is also true for the diagnosis of a 365 great amount of medical conditions: the descriptions of symptoms are historically largely based on data coming from male participants, leading to increased likelihood of misdiagnosis in females [1]. As Shansky and Murphy recently advocated, representing both sexes instead of just one is a more rigorous approach that can improve reproducibility and translatability, potentially allowing for the discovery of "sex-dependent mechanisms underlying a common outcome” [1]. 


\section{Materials and Methods}

This study was reviewed and approved by the Ethics Committees of the Humboldt-Universität zu Berlin (approval code HU-KSBF-EK_2018_0013), Kassel University (E05201602) and Heidelberg University (AZ Schw 2018 1/2). All the participants gave written informed consent for

375 the experimental procedure, in accordance with the Declaration of Helsinki.

Experimental protocols

We recruited 215 healthy volunteers, 70 of which were of older age (65 to 84 years). The young adults (20 to 43 years) were asked to walk and/or run on a treadmill, while the older adults were only asked to perform treadmill walking. Three experimental conditions were obtained as follows:

380 walking, 70 young adults (henceforth G1, 35 females); walking, 70 older adults (henceforth G2, 35 females); running, 120 young adults (henceforth G3, 60 females; some participants in G3 were also included in G1, the specifics are given in the supplementary information on Zenodo (DOI: 10.5281/zenodo.5171754). Details about the participants and locomotion speeds are given in Table 1. The participants completed a self-selected warm-up walking (G1, G2) or running (G1, G3) on

385 a treadmill, typically lasting between 3 and 5 min [56]. The participants were asked to walk or run at either their preferred speed, found through the method of limits [57], or at a fixed speed chosen to be close to the average preferred speed, depending on the group [56,58] (details are given in the supplementary information on Zenodo; DOI: 10.5281/zenodo.5171754). The treadmills used were of three different types, depending on the location of the recordings: a) mercury, H-p-cosmos

390 Sports \& Medical GmbH, Nussdorf, Germany equipped with a pressure plate recording the plantar pressure distribution at $120 \mathrm{~Hz}$ (FDM- THM-S, zebris Medical GmbH, Isny im Allgäu, Germany) for the experiments conducted at the Humboldt-Universität zu Berlin; b) Laufergotest, Erich Jäger, 
Würzburg, Germany for the experiments at Kassel University; c) BalanceTutor ${ }^{\mathrm{TM}}$, MediTouch LTD,

Netanya, Israel, for the experiments at Heidelberg University.

Table 1 Participant data and group division. Values are presented as mean \pm standard deviation.

\begin{tabular}{|c|c|c|c|c|c|c|c|}
\hline Group & Task & Sex & $\mathrm{N}$ & Age [years] & Height $[\mathrm{cm}]$ & Mass [kg] & Speed $[\mathrm{m} / \mathrm{s}]$ \\
\hline \multirow{2}{*}{ G1 } & \multirow{4}{*}{$\begin{array}{c}\text { Treadmill } \\
\text { walking }\end{array}$} & $\mathrm{F}$ & 35 & $25.5 \pm 3.5$ & $167.5 \pm 6.6$ & $60.0 \pm 8.1$ & $1.27 \pm 0.12$ \\
\hline & & $\mathrm{M}$ & 35 & $28.3 \pm 4.3$ & $178.6 \pm 6.1$ & $75.6 \pm 9.4$ & $1.30 \pm 0.11$ \\
\hline \multirow{2}{*}{ G2 } & & $\mathrm{F}$ & 35 & $71.4 \pm 4.9$ & $165.4 \pm 6.6$ & $65.3 \pm 9.0$ & $1.11 \pm 0.01$ \\
\hline & & $\mathrm{M}$ & 35 & $73.3 \pm 4.5$ & $176.7 \pm 6.1$ & $79.2 \pm 10.4$ & $1.13 \pm 0.07$ \\
\hline \multirow{2}{*}{ G3 } & \multirow{2}{*}{$\begin{array}{c}\text { Treadmill } \\
\text { running }\end{array}$} & $\mathrm{F}$ & 60 & $28.5 \pm 4.9$ & $167.7 \pm 6.3$ & $61.0 \pm 7.0$ & $2.57 \pm 0.28$ \\
\hline & & $\mathrm{M}$ & 60 & $30.5 \pm 5.2$ & $180.7 \pm 6.0$ & $76.2 \pm 9.4$ & $2.68 \pm 0.31$ \\
\hline
\end{tabular}

\section{$\underline{\text { EMG recordings }}$}

The muscle activity of the following 13 muscles of the right side was recorded in all groups:

400 gluteus medius, gluteus maximus, tensor fascice late, rectus femoris, vastus medialis, vastus lateralis, semitendinosus, biceps femoris (long head), tibialis anterior, peroneus longus, gastrocnemius medialis, gastrocnemius lateralis and soleus. The electrodes were positioned as previously reported and following SENIAM (surface EMG for a non-invasive assessment of muscles) recommendations [58,59]. After around $60 \mathrm{~s}$ habituation [47], we recorded two trials of 
16-channel wireless bipolar EMG system (Wave Plus wireless EMG with PicoEMG sensors, Cometa srl, Bareggio, Italy). For the EMG recordings, we used foam-hydrogel electrodes with snap connector (H124SG, Medtronic plc, Dublin, Ireland). The first 30 gait cycles of the recorded trial were considered for subsequent analysis [60]. All the recordings can be downloaded from the 410 supplementary data set, accessible on Zenodo (DOI: 10.5281/zenodo.5171754).

\section{Gait cycle parameters}

The gait cycle segmentation (foot touchdown and lift-off timing) was obtained by the elaboration of the data acquired by either the pressure plate integrated in one of the treadmills (3 participants in G1 and 11 in G3), a foot-mounted 3D accelerometer (PicoEMG, Cometa srl, Bareggio, Italy)

415 recording at $142.85 \mathrm{~Hz}$ (49 participants in G1, all participants in G2 and 94 participants of G3) or 3D kinematics of the foot recorded via a six-camera motion capture system (Oqus 3+, Qualisys $\mathrm{AB}$, Gothenburg, Sweden) acquiring at $300 \mathrm{~Hz}$ (18 participants in G1 and 15 in G3). For the detection of both touchdown and lift-off of the foot, we used externally-validated algorithms based on foot acceleration data. Specifically, for touchdown we adopted the modified foot contact 420 algorithm [61], which uses a characteristic peak in the vertical acceleration to determine touchdown. For lift-off, we searched for a characteristic peak in the longitudinal (i.e., anteroposterior) acceleration of the foot between two consecutive touchdowns. Both algorithms were validated against data recorded from 30 young adults (15 females, height $173 \pm 10 \mathrm{~cm}$, body mass $68 \pm 12 \mathrm{~kg}$, age $28 \pm 5$ years) during walking and running (at $1.4 \mathrm{~m} / \mathrm{s}$ and $2.8 \mathrm{~m} / \mathrm{s}$,

425 respectively) over a 900 x 600 mm force plate (1 kHz, AMTI BP600, Advanced Mechanical Technology, Inc., Watertown, MA, USA), with ten steps recorded per participants, for a total of 300 steps for walking and 300 for running. For the 14 participants in which plantar pressure distribution was recorded, we elaborated this data to obtain the gait cycle segmentation [56]. Gait 
430 were the cadence (i.e., number of steps per minute), stance and swing times.

\section{$\underline{\text { Muscle synergies }} \underline{\text { extraction }}$}

Muscle synergies data were extracted from the recorded EMG activity through a custom script (R v4.0.4, R Core Team, 2021, R Foundation for Statistical Computing, Vienna, Austria) using the classical Gaussian NMF algorithm [62], the application of which was extensively described in the

435 past $[46,47,63,64]$. Briefly, we high-pass filtered, full-wave rectified and lastly low-pass filtered the raw EMG signals using a $4^{\text {th }}$ order IIR Butterworth zero-phase filter with cut-off frequencies $50 \mathrm{~Hz}$ (high-pass) and $20 \mathrm{~Hz}$ (low-pass). After amplitude normalization of each muscle to the maximum of each trial, gait cycles were time-normalized to 200 points each (100 points for the stance, 100 points for the swing phase). Synergies were then extracted through NMF and the

440 quality of reconstruction assessed with the coefficient of determination $\mathrm{R}^{2}$. Similar time-dependent motor primitives [38,58] and time-invariant motor modules [58,65] were then functionally classified through a clustering approach based on NMF [41]. The source code for the pre-processing of raw EMG data and for the extraction and classification of muscle synergies is available at https://github.com/alesantuz/musclesyneRgies [66]. Version 0.7.1-alpha, used in this paper, is archived 445 at Zenodo (DOI: 10.5281/zenodo.5171754).

\section{$\underline{\text { Coactivation index }}$}

To obtain the ratio of flexor and extensor muscle contribution to each joint in a specific synergy, we calculated the coactivation index (CaI) from the motor modules as follows. For every synergy and trial, we calculated the mean contributions of the flexors and those of the extensors. For the

450 hip, we considered the FL and RF as flexors and the ME and MA as extensors. For the knee, the flexors were the ST and BF and the extensors the RF, VM and VL. For the ankle, the only flexor 
(i.e., foot dorsiflexor) was the TA and the extensors (i.e., foot plantarflexors) the PL, GM, GL and SO. For each joint, the mean of the flexor contributions $\overline{f l e x}$ and the mean of the extensor contributions $\overline{e x t}$ were forced to sum to $1: C a I=\overline{f l e x} /(\overline{f l e x}+\overline{e x t})$. Following this definition:

455 a) $\mathrm{CaI}=0$ when only extensors are contributing to the considered joint; b) $\mathrm{CaI}=1$ when only flexors are giving their contribution; c) $\mathrm{CaI}=0.5$ if flexors and extensors are equally contributing (i.e., full coactivation of flexors and extensors).

Linear and nonlinear metrics for the analysis of motor primitives

As linear metrics for the comparison of motor primitives we used the full width at half maximum 460 and the center of activity. The full width at half maximum describes the duration or width of motor primitives and it was calculated cycle-by-cycle after subtracting the cycle's minimum as the number of points exceeding each cycle's half maximum, and then averaged [43]. The center of activity, an indication of when the main activation is happening in time, was also calculated cycleby-cycle as the angle of the vector in polar coordinates that points to the center of mass of the 465 circular distribution defined between 0 (touchdown) and 360 (next touchdown) and then averaged [45].

To assess the local and global complexity of motor primitives, we calculated the Higuchi's fractal dimension and Hurst exponent, respectively [67,68]. The local complexity can be seen as a measure of "roughness" (or noise content) in the signal within each gait cycle, while the global 470 complexity is a measure of how stereotyped each cycle's activations are, when compared to the others. The numerical procedures for obtaining both metrics were recently reported in detail [69]. The Higuchi's fractal dimension was calculated with a maximum window size of 10 points, while the Hurst exponent was obtained by imposing a minimum window length of 200 points, equal to the length of a time-normalized gait cycle. Values of the Higuchi's fractal dimension range from 
4751 to 2, with increasing values correlating to increasingly complex (or rough) data and 1.5 indicating random Gaussian noise [67,70,71]. The Hurst exponent can vary between 0 and 1 . For $0.5<$ Hurst exponent $<1$, in the long-term high values in the time series (the motor primitive in our case) will be probably followed by other high values and a positive or negative trend is visible [72,73]. For $0<$ Hurst exponent $<0.5$, in the long term high values in the series will be probably followed by

480 low values, with a frequent switch between high and low values [72,73] and values moving from 0.5 towards 0 indicating increasingly stereotyped gait cycle’s activity (e.g., a sinusoidal wave has Hurst exponent $=0$ and each cycle is identical to the others). A completely random series will have Hurst exponent $=0.5[73,74]$.

\section{$\underline{\text { Statistics }}$}

485 To investigate the effects of sex on the modular muscle activation, we followed a Bayesian multilevel modeling approach implemented in the R package brms 2.15.0 [75,76]. We adopted the Bayesian framework in an effort to move away from the concept of statistical significance and its dichotomous nature, including in our analysis prior data and available information for computing credible intervals around the observed effects [77,78]. Credible intervals are "the Bayesian analogue of a classical

490 confidence interval" [79], with the advantage that it is possible to make probability statements on them: a 95\% credible intervals can be defined as that interval around the point estimate having a 95\% probability of encompassing the population value, given the data and the prior assumptions [79]. For each dependent variable of interest, we built a mixed effects model containing both “fixed” and "random” effects. The constant effects analyzed were: sex, age and their interaction in G1 and

495 G2 (Eq. 1); sex in G3 (Eq. 2). Locomotion speed was modeled as a covariate in all groups, to account for the different speeds between groups and/or participants. As random effects, we 
considered by-participant random slopes for the effect of the independent variables. The two models can be respectively summarized with the following equations:

$$
y \sim \operatorname{speed}+\operatorname{sex} * \text { age }+(1 \mid \text { participant })(1)
$$

$$
y \sim \text { speed }+ \text { sex }+(1 \mid \text { participant })(2)
$$

where $y$ is the analyzed dependent variable. Each model was run with five independent Markov chains of 5000 iterations, with the first 2000 warm-up iterations used only for calibration and then discarded, thus resulting in 15000 post-warm-up samples. Sex was contrast-coded symmetrically (female = 0.5, male $=-0.5)$. Convergence of the chains and sufficient sampling of posterior distributions were 505 confirmed by ensuring a potential scale reduction factor $\hat{R}<1.01$ and an effective sample size of at least $20 \%$ of the number of iterations [80]. Posterior distributions were summarized by the following estimators: mean, standard error and the lower and upper bounds of the 95\% credible intervals. We used different priors depending on the investigated parameters and based on previously published data, without distinction of sex or age. Specifically, we used a gamma prior for the factorization 510 rank with parameters shape and scale: rank $\sim \Gamma(9,0.5)$. For all the other parameters, we used normal priors with the following means and standard deviations: stance duration walking N(700 ms, $100 \mathrm{~ms})$; stance duration running N(300 ms, $80 \mathrm{~ms})$; swing duration walking N(400 ms, $70 \mathrm{~ms})$; swing duration running N(450 ms, $100 \mathrm{~ms})$; cadence walking N(110 steps/min, 15 steps/min); cadence running N(165 steps/min, 25 steps/min); percentage of combined synergies

$515 \sim \mathrm{N}(20 \%, 10 \%) ; \mathrm{R}^{2} \sim \mathrm{N}(30,15) ; \mathrm{FWHM} \sim \mathrm{N}(30$ points, 15 points $) ;$ CoA N(100 points, 35 points); Higuchi's fractal dimension $\sim \mathrm{N}(0.30,0.15)$; Hurst exponent $\sim \mathrm{N}(1.3,0.2)$; CaI $\sim \mathrm{N}(30 \%$, $15 \%)$. In the results section we reported the slope estimated by the statistical model as $\beta$ and the estimated 95\% credible interval as CrI. To report the effect size, we calculated the Bayesian equivalent of the Cohen's $d$, called $\delta_{\mathrm{t}}$ [79]. Moreover, we expressed a Bayesian version of the $\mathrm{R}^{2}$, 
520 here called $\mathrm{R}^{2}$ Bayes and calculated as the variance of the predicted values divided by the variance of predicted values plus the expected variance of the errors, to quantify the model's fit to the original data, [81]. Values of $\delta_{\mathrm{t}}$ and $\mathrm{R}^{2}$ Bayes are to be interpreted similarly to the Cohen's $d$ and the classical $\mathrm{R}^{2}$, respectively. 
Acknowledgments: The authors are grateful to all the participants and acknowledge support by the German Research Foundation (DFG) and the Open Access Publication Fund of the HumboldtUniversität zu Berlin. The authors disclose any professional relationship with companies or manufacturers who might benefit from the results of the present study. 


\section{Figure captions}

Figure 1. Gait temporal parameters. Boxplots describing the stance and swing duration and the cadence for the three groups. Raw data points and their density estimates are presented to the right side of each boxplot.

Figure 2. Average electromyographic activity of lower limb muscles. Average EMG activity of the recorded muscles in the three groups. The x-axis full scale represents the averaged EMG activity over the gait cycle (with stance and swing normalized to the same amount of points and divided by a vertical line) and the y-axis the amplitude normalized to the maximum mean activity for each muscle. Muscle abbreviations: $\mathrm{ME}=$ gluteus medius, $\mathrm{MA}=$ gluteus maximus, $\mathrm{FL}=$ tensor

540 fascice latce, $\mathrm{RF}=$ rectus femoris, $\mathrm{VM}=$ vastus medialis, $\mathrm{VL}=$ vastus lateralis, $\mathrm{ST}=$ semitendinosus, $\mathrm{BF}=$ biceps femoris, $\mathrm{TA}=$ tibialis anterior, $\mathrm{PL}=$ peroneus longus, $\mathrm{GM}=$ gastrocnemius medialis, $\mathrm{GL}=$ gastrocnemius lateralis, $\mathrm{SO}=$ soleus.

Figure 3. Muscle synergies for human walking and running. Motor modules and motor primitives of the four fundamental synergies for human walking (young and older females and 545 males) and running (young females and males). The motor modules are presented on a normalized y-axis base and each muscle contribution within one synergy can range from 0 to 1 . Each bar represents the average of the individual trials, shown as dots. For the motor primitives, the x-axis full scale represents the averaged activation pattern over one gait cycle (with stance and swing normalized to the same amount of points and divided by a vertical line) and the $y$-axis the 550 normalized amplitude. A standard deviation band represents variability across participants. Muscle abbreviations: $\mathrm{ME}=$ gluteus medius, $\mathrm{MA}=$ gluteus maximus, $\mathrm{FL}=$ tensor fascice late, $\mathrm{RF}=$ rectus femoris, $\mathrm{VM}=$ vastus medialis, $\mathrm{VL}=$ vastus lateralis, $\mathrm{ST}=$ semitendinosus, $\mathrm{BF}=$ biceps femoris, 
TA=tibialis anterior, $\mathrm{PL}=$ peroneus longus, $\mathrm{GM}=$ gastrocnemius medialis, $\mathrm{GL}=$ gastrocnemius lateralis, $\mathrm{SO}=$ soleus.

555 Figure 4. Overlay of muscle synergies for human locomotion and coactivation index. Panel a: average motor modules and motor primitives of the four fundamental synergies for human walking (young and older females and males) and running (young females and males). Synergies from females and males are overlaid for comparison. The motor modules are presented on a normalized y-axis base and each muscle contribution within one synergy can range from 0 (minimum muscle

560 contribution to each synergy and sex) to 1 (maximum muscle contribution). Each bar represents the average of the individual trials. For the motor primitives, the $\mathrm{x}$-axis full scale represents the averaged activation pattern of all trials for each locomotion condition and sex over one gait cycle (with stance and swing normalized to the same amount of points and divided by a vertical line) and the $\mathrm{y}$-axis the normalized amplitude. Muscle abbreviations: $\mathrm{ME}=$ gluteus medius, $\mathrm{MA}=$ gluteus

565 maximus, $\mathrm{FL}=$ tensor fascice latce, $\mathrm{RF}=$ rectus femoris, $\mathrm{VM}=$ vastus medialis, $\mathrm{VL}=$ vastus lateralis, $\mathrm{ST}=$ semitendinosus, $\mathrm{BF}=$ biceps femoris, $\mathrm{TA}=$ tibialis anterior, $\mathrm{PL}=$ peroneus longus, $\mathrm{GM}=$ gastrocnemius medialis, $\mathrm{GL}=$ gastrocnemius lateralis, $\mathrm{SO}=$ soleus. Panel b: average coactivation index (CoI) calculated on the motor modules. Arrows pointing to the left $(\mathrm{CoI}=0)$ represent pure extensor contribution, while arrows pointing to the right $(\mathrm{CoI}=1)$ translate to pure 570 extensor contribution. Arrows pointing up $(\mathrm{CoI}=0.5)$ indicate perfect balance between extensor and flexor contribution, or perfect coactivation.

Figure 5. Center of activity (CoA) and full width at half maximum (FWHM) of motor primitives. Panel a: boxplots describing the CoA of motor primitives for the three groups. Raw data points and their density estimates are presented to the right side of each boxplot. Panel b: 575 similar to panel a, but for the FWHM. 
Figure 6. Each participant's motor primitives and fractal analysis outcomes. Panel a: the averaged motor primitives over one gait cycle are presented for each participant in thin lines, the global mean curves being the thick lines. Panel b: boxplots describing the Higuchi’s fractal dimension (HFD) and Hurst exponent $(\mathrm{H})$ of motor primitives for the three groups. Raw data points

580 and their density estimates are presented to the right side of each boxplot. HFD and H are measures of local and global complexity, respectively. 


\section{References}

1. Shansky RM, Murphy AZ. Considering sex as a biological variable will require a global shift in science culture. Nat Neurosci. 2021. doi:10.1038/s41593-021-00806-8

2. Woitowich NC, Beery AK, Woodruff TK. A 10-year follow-up study of sex inclusion in the biological sciences. Elife. 2020;9: 1-8. doi:10.7554/eLife.56344

3. Beery AK, Zucker I. Sex bias in neuroscience and biomedical research. Neurosci Biobehav Rev. 2011;35: 565-572. doi:10.1016/j.neubiorev.2010.07.002

590 4. Ansdell P, Thomas K, Hicks KM, Hunter SK, Howatson G, Goodall S. Physiological sex differences affect the integrative response to exercise: Acute and chronic implications. Exp Physiol. 2020; 2007-2021. doi:10.1113/EP088548

5. Tannenbaum C, Ellis RP, Eyssel F, Zou J, Schiebinger L. Sex and gender analysis improves science and engineering. Nature. 2019;575: 137-146. doi:10.1038/s41586-019-1657-6

595 6. Bernstein NA. The co-ordination and regulation of movements. Pergamon Press. Oxford: Pergamon Press Ltd.; 1967.

7. Rowe E, Beauchamp MK, Astephen Wilson J. Age and sex differences in normative gait patterns. Gait Posture. 2021;88: 109-115. doi:10.1016/j.gaitpost.2021.05.014

8. Bruening DA, Frimenko RE, Goodyear CD, Bowden DR, Fullenkamp AM. Sex differences in whole body gait kinematics at preferred speeds. Gait Posture. 2015;41: 540-545. doi:10.1016/j.gaitpost.2014.12.011

9. Kerrigan DC, Todd MK, Croce UD. GENDER DIFFERENCES IN JOINT BIOMECHANICS DURING WALKING Normative Study in Young Adults. Am J Phys 
Med Rehabil. 1998;77: 2-7. doi:10.1097/00002060-199801000-00002

605 10. Ko S uk, Tolea MI, Hausdorff JM, Ferrucci L. Sex-specific differences in gait patterns of healthy older adults: Results from the Baltimore Longitudinal Study of Aging. J Biomech. 2011;44: 1974-1979. doi:10.1016/j.jbiomech.2011.05.005

11. Frimenko R, Goodyear C, Bruening D. Interactions of sex and aging on spatiotemporal metrics in non-pathological gait: A descriptive meta-analysis. Physiother (United Kingdom). 2015;101: 266-272. doi:10.1016/j.physio.2015.01.003

12. da Fonseca ST, Vaz DV, de Aquino CF, Brício RS. Muscular co-contraction during walking and landing from a jump: Comparison between genders and influence of activity level. J Electromyogr Kinesiol. 2006;16: 273-280. doi:10.1016/j.jelekin.2005.07.005

13. Silder A, Delp SL, Besier T. Men and women adopt similar walking mechanics and muscle activation patterns during load carriage. J Biomech. 2013;46: 2522-2528. doi:10.1016/j.jbiomech.2013.06.020

14. Anders C, Patenge S, Sander K, Layher F, Biedermann U, Kinne RW. Detailed spatial characterization of superficial hip muscle activation during walking: A multi-electrode surface EMG investigation of the gluteal region in healthy older adults. PLoS One. 2017;12: 1-24. doi:10.1371/journal.pone.0178957

15. Di Nardo F, Mengarelli A, Strazza A, Agostini V, Knaflitz M, Burattini L, et al. A new parameter for quantifying the variability of surface electromyographic signals during gait: The occurrence frequency. J Electromyogr Kinesiol. 2017;36: 25-33. doi:10.1016/j.jelekin.2017.06.006 
625 16. Chandran VD, Calalo JA, Dixon PC, Dennerlein JT, Schiffman JM, Pal S. Knee muscle cocontractions are greater in old compared to young adults during walking and stair use. Gait Posture. 2019;73: 315-322. doi:10.1016/j.gaitpost.2019.07.501

17. Wall R, Garcia G, Läubli T, Seibt R, Rieger MA, Martin B, et al. Physiological changes during prolonged standing and walking considering age, gender and standing work experience. Ergonomics. 2020;63: 579-592. doi:10.1080/00140139.2020.1725145

18. Gorsuch J, Long J, Miller K, Primeau K, Rutledge S, Sossong A, et al. The Effect of Squat Depth on Multiarticular Muscle Activation in Collegiate Cross-Country Runners. J Strength Cond Res. 2013;27: 2619-2625. doi:10.1519/JSC.0b013e31828055d5

19. DeMont RG, Lephart SM. Effect of sex on preactivation of the gastrocnemius and hamstring muscles. Br J Sports Med. 2004;38: 120-124. doi:10.1136/bjsm.2002.000195

20. Chumanov ES, Wall-Scheffler C, Heiderscheit BC. Gender differences in walking and running on level and inclined surfaces. Clin Biomech. 2008;23: 1260-1268. doi:10.1016/j.clinbiomech.2008.07.011

21. Chung MJ, Wang MJJ. The change of gait parameters during walking at different percentage of preferred walking speed for healthy adults aged 20-60 years. Gait Posture. 2010;31: 131-135. doi:10.1016/j.gaitpost.2009.09.013

22. Bailey CA, Corona F, Pilloni G, Porta M, Fastame MC, Hitchcott PK, et al. Sex-dependent and sex-independent muscle activation patterns in adult gait as a function of age. Exp Gerontol. 2018;110: 1-8. doi:10.1016/j.exger.2018.05.005

645 23. Willson JD, Petrowitz I, Butler RJ, Kernozek TW. Male and female gluteal muscle activity 
and lower extremity kinematics during running. Clin Biomech. 2012;27: 1052-1057. doi:10.1016/j.clinbiomech.2012.08.008

24. Moltó IN, Albiach JP, Amer-Cuenca JJ, Segura-Ortí E, Gabriel W, Martínez-Gramage J. Wearable Sensors Detect Differences between the Sexes in Lower Limb Electromyographic Activity and Pelvis 3D Kinematics during Running. Sensors. 2020;20: 6478. doi:10.3390/s20226478

25. Aljohani M, Kipp K. Use of self-organizing maps to study sex- and speed-dependent changes in running biomechanics. Hum Mov Sci. 2020;72: 102649. doi:10.1016/j.humov.2020.102649

26. Baur H, Hirschmüller A, Cassel M, Müller S, Mayer F. Gender-specific neuromuscular activity of the M. peroneus longus in healthy runners - A descriptive laboratory study. Clin Biomech. 2010;25: 938-943. doi:10.1016/j.clinbiomech.2010.06.009

27. Mengarelli A, Maranesi E, Burattini L, Fioretti S, Di Nardo F. Co-contraction activity of ankle muscles during walking: A gender comparison. Biomed Signal Process Control. 2017;33: 1-9. doi:10.1016/j.bspc.2016.11.010

28. Smith SL, Woodburn J, Steultjens MPM. Sex- and osteoarthritis-related differences in muscle co-activation during weight-bearing tasks. Gait Posture. 2020;79: 117-125. doi:10.1016/j.gaitpost.2020.04.019

29. Tirosh O, Sparrow WA. Age and walking speed effects on muscle recruitment in gait termination. Gait Posture. 2005;21: 279-288. doi:10.1016/j.gaitpost.2004.03.002

30. Dewolf AH, Sylos-Labini F, Cappellini G, Ivanenko YP, Lacquaniti F. Age-related changes 
in the neuromuscular control of forward and backward locomotion. Masani K, editor. PLoS One. 2021;16: e0246372. doi:10.1371/journal.pone.0246372

31. Santuz A, Brüll L, Ekizos A, Schroll A, Eckardt N, Kibele A, et al. Neuromotor Dynamics of Human Locomotion in Challenging Settings. iScience. 2020;23: 100796. doi:10.1016/j.isci.2019.100796

32. Kwee-Meier ST, Mertens A, Jeschke S. Age-induced changes in the lower limb muscle activities during uphill walking at steep grades. Gait Posture. 2018;62: 490-496. doi:10.1016/j.gaitpost.2018.04.003

675 33. Monaco V, Ghionzoli A, Micera S. Age-Related Modifications of Muscle Synergies and Spinal Cord Activity During Locomotion. J Neurophysiol. 2010;104: 2092-2102. doi:10.1152/jn.00525.2009

34. McGibbon CA, Krebs DE. Discriminating age and disability effects in locomotion: Neuromuscular adaptations in musculoskeletal pathology. J Appl Physiol. 2004;96: 149_ 160. doi:10.1152/japplphysiol.00422.2003

35. Bizzi E, Mussa-Ivaldi FA, Giszter SF. Computations underlying the execution of movement: a biological perspective. Science (80- ). 1991;253: 287-291. doi:10.1126/science.1857964

36. Tresch MC, Saltiel P, Bizzi E. The construction of movement by the spinal cord. Nat Neurosci. 1999;2: 162-167. doi:10.1038/5721

37. Cappellini G, Ivanenko YP, Poppele RE, Lacquaniti F. Motor patterns in human walking and running. J Neurophysiol. 2006;95: 3426-37. doi:10.1152/jn.00081.2006 
38. Dominici N, Ivanenko YP, Cappellini G, D’Avella A, Mondi V, Cicchese M, et al. Locomotor Primitives in Newborn Babies and Their Development. Science (80- ). 2011;334: 997-999. doi:10.1126/science.1210617

39. Ting LH, Chiel HJ, Trumbower RD, Allen JL, McKay JL, Hackney ME, et al. Neuromechanical Principles Underlying Movement Modularity and Their Implications for Rehabilitation. Neuron. 2015;86: 38-54. doi:10.1016/j.neuron.2015.02.042

40. Chiu MC, Wang MJ. The effect of gait speed and gender on perceived exertion, muscle activity, joint motion of lower extremity, ground reaction force and heart rate during normal walking. Gait Posture. 2007;25: 385-392. doi:10.1016/j.gaitpost.2006.05.008

41. Mileti I, Serra A, Wolf N, Munoz-Martel V, Ekizos A, Palermo E, et al. Muscle Activation Patterns Are More Constrained and Regular in Treadmill Than in Overground Human Locomotion. Front Bioeng Biotechnol. 2020;8. doi:10.3389/fbioe.2020.581619

700 42. Lacquaniti F, Ivanenko YP, Zago M. Patterned control of human locomotion. J Physiol. 2012;590: 2189-2199. doi:10.1113/jphysiol.2011.215137

43. Martino G, Ivanenko YP, Serrao M, Ranavolo A, D’Avella A, Draicchio F, et al. Locomotor patterns in cerebellar ataxia. J Neurophysiol. 2014;112: 2810-2821. doi:10.1152/jn.00275.2014

705 44. Janshen L, Santuz A, Ekizos A, Arampatzis A. Fuzziness of muscle synergies in patients with multiple sclerosis indicates increased robustness of motor control during walking. Sci Rep. 2020;10: 7249. doi:10.1038/s41598-020-63788-w

45. Cappellini G, Ivanenko YP, Martino G, MacLellan MJ, Sacco A, Morelli D, et al. Immature 
Spinal Locomotor Output in Children with Cerebral Palsy. Front Physiol. 2016;7: 1-21. doi:10.3389/fphys.2016.00478

46. Santuz A, Akay T, Mayer WP, Wells TL, Schroll A, Arampatzis A. Modular organization of murine locomotor pattern in the presence and absence of sensory feedback from muscle spindles. J Physiol. 2019;597: 3147-3165. doi:10.1113/JP277515

47. Santuz A, Ekizos A, Eckardt N, Kibele A, Arampatzis A. Challenging human locomotion: stability and modular organisation in unsteady conditions. Sci Rep. 2018;8: 2740. doi:10.1038/s41598-018-21018-4

48. Santuz A, Laflamme OD, Akay T. Differential role of spinal and supraspinal processing of proprioceptive information in mouse locomotion. bioRxiv. 2021. Available: https://www.biorxiv.org/content/10.1101/2021.04.28.441796v1

720 49. Martino G, Ivanenko YP, D’Avella A, Serrao M, Ranavolo A, Draicchio F, et al. Neuromuscular adjustments of gait associated with unstable conditions. J Neurophysiol. 2015;114: 2867-2882. doi:10.1152/jn.00029.2015

50. Santuz A, Ekizos A, Kunimasa Y, Kijima K, Ishikawa M, Arampatzis A. Lower complexity of motor primitives ensures robust control of high-speed human locomotion. Heliyon. 2020;6: e05377. doi:10.1016/j.heliyon.2020.e05377

51. Daniels JT, Daniels N. Running economy of elite male and elite female runners. Med Sci Sport Exerc. 1992;24: 483-489.

52. Glace BW, McHugh MP, Gleim GW. Effects of a 2-hour run on metabolic economy and lower extremity strength in men and women. J Orthop Sports Phys Ther. 1998;27: 189-196. 
53. Ansdell P, Škarabot J, Atkinson E, Corden S, Tygart A, Hicks KM, et al. Sex differences in fatigability following exercise normalised to the power-duration relationship. J Physiol. 2020;598: 5717-5737. doi:10.1113/JP280031

54. Temesi J, Arnal PJ, Rupp T, Féasson L, Cartier R, Gergelé L, et al. Are females more resistant to extreme neuromuscular fatigue? Med Sci Sports Exerc. 2015;47: 1372-1382. doi:10.1249/MSS.0000000000000540

55. Glace BW, Kremenic IJ, McHugh MP. Sex differences in central and peripheral mechanisms of fatigue in cyclists. Eur J Appl Physiol. 2013;113: 1091-1098. doi:10.1007/s00421-012-2516-4

740 56. Santuz A, Ekizos A, Arampatzis A. A Pressure Plate-Based Method for the Automatic Assessment of Foot Strike Patterns During Running. Ann Biomed Eng. 2016;44: 16461655. doi:10.1007/s10439-015-1484-3

57. Treutwein B. Adaptive psychophysical procedures. Vision Res. 1995;35: 2503-2522. doi:10.1016/0042-6989(95)00016-S

745 58. Santuz A, Ekizos A, Janshen L, Baltzopoulos V, Arampatzis A. On the Methodological Implications of Extracting Muscle Synergies from Human Locomotion. Int J Neural Syst. 2017;27: 1750007. doi:10.1142/S0129065717500071

59. Hermens HJ, Freriks B, Disselhorst-Klug C, Rau G. Development of recommendations for SEMG sensors and sensor placement procedures. J Electromyogr Kinesiol. 2000;10: 36174. Available: http://www.ncbi.nlm.nih.gov/pubmed/11018445 
60. Santuz A, Ekizos A, Janshen L, Mersmann F, Bohm S, Baltzopoulos V, et al. Modular Control of Human Movement During Running: An Open Access Data Set. Front Physiol. 2018;9: 1509. doi:10.3389/fphys.2018.01509

61. Maiwald C, Sterzing T, Mayer TA, Milani TL. Detecting foot-to-ground contact from kinematic data in running. Footwear Sci. 2009;1: 111-118. doi:10.1080/19424280903133938

62. Lee DD, Seung HS. Learning the parts of objects by non-negative matrix factorization. Nature. 1999;401: 788-91. doi:10.1038/44565

63. D’Avella A, Bizzi E. Shared and specific muscle synergies in natural motor behaviors. Proc Natl Acad Sci U S A. 2005;102: 3076-81. doi:10.1073/pnas.0500199102

64. Cheung VC-K, D’Avella A, Tresch MC, Bizzi E. Central and sensory contributions to the activation and organization of muscle synergies during natural motor behaviors. J Neurosci. 2005;25: 6419-34. doi:10.1523/JNEUROSCI.4904-04.2005

65. Gizzi L, Nielsen JF, Felici F, Ivanenko YP, Farina D. Impulses of activation but not motor modules are preserved in the locomotion of subacute stroke patients. J Neurophysiol. 2011;106: 202-210. doi:10.1152/jn.00727.2010

66. Santuz A. musclesyneRgies v0.7.1-alpha. GitHub; 2021. Available: https://github.com/alesantuz/musclesyneRgies/releases/tag/v0.7.1-alpha

67. Higuchi T. Approach to an irregular time series on the basis of the fractal theory. Phys D Nonlinear Phenom. 1988;31: 277-283. doi:10.1016/0167-2789(88)90081-4

68. Hurst HE. Long-term storage capacity of reservoirs. Trans Am Soc Civ Eng. 1951;116: 
770-808.

69. Santuz A, Akay T. Fractal analysis of muscle activity patterns during locomotion: pitfalls and how to avoid them. J Neurophysiol. 2020;124: 1083-1091. doi:10.1152/jn.00360.2020

775 70. Anmuth CJ, Goldberg G, Mayer NH. Fractal dimension of electromyographic signals recorded with surface electrodes during isometric contractions is linearly correlated with muscle activation. Muscle Nerve. 1994;17: 953-954. doi:10.1002/mus.880170819

71. Kesić S, Spasić SZ. Application of Higuchi’s fractal dimension from basic to clinical neurophysiology: A review. Comput Methods Programs Biomed. 2016;133: 55-70. doi:10.1016/j.cmpb.2016.05.014

72. Gneiting T, Schlather M. Stochastic Models That Separate Fractal Dimension and the Hurst Effect. SIAM Rev. 2004;46: 269-282. doi:10.1137/S0036144501394387

73. Mandelbrot BB. The Fractal Geometry of Nature. New York: W. H. Freeman and Co.; 1983.

74. Qian B, Rasheed K. Hurst exponent and financial market predictability. Proceedings of the Second IASTED International Conference on Financial Engineering and Applications. 2004. pp. 203-209.

75. Bürkner PC. brms: An R package for Bayesian multilevel models using Stan. J Stat Softw. 2017;80. doi:10.18637/jss.v080.i01

76. Carpenter B, Gelman A, Hoffman MD, Lee D, Goodrich B, Betancourt M, et al. Stan: A probabilistic programming language. J Stat Softw. 2017;76. doi:10.18637/jss.v076.i01

77. Amrhein V, Greenland S, McShane B. Scientists rise up against statistical significance. Nature. 2019;567: 305-307. doi:10.1038/d41586-019-00857-9 
78. Wasserstein RL, Schirm AL, Lazar NA. Moving to a World Beyond “p < 0.05.” Am Stat. 2019;73: 1-19. doi:10.1080/00031305.2019.1583913

795 79. Nalborczyk L, Batailler C, Loevenbruck H, Vilain A, Bürkner PC. An introduction to bayesian multilevel models using brms: A case study of gender effects on vowel variability in standard Indonesian. J Speech, Lang Hear Res. 2019;62: 1225-1242. doi:10.1044/2018_JSLHR-S-18-0006

80. Dainese M, Martin EA, Aizen MA, Albrecht M, Bartomeus I, Bommarco R, et al. A global synthesis reveals biodiversity-mediated benefits for crop production. Sci Adv. 2019;5: eaax0121. doi:10.1126/sciadv.aax0121

81. Gelman A, Goodrich B, Gabry J, Vehtari A. R-squared for Bayesian Regression Models. Am Stat. 2019;73: 307-309. doi:10.1080/00031305.2018.1549100 


\section{Supplementary figures}

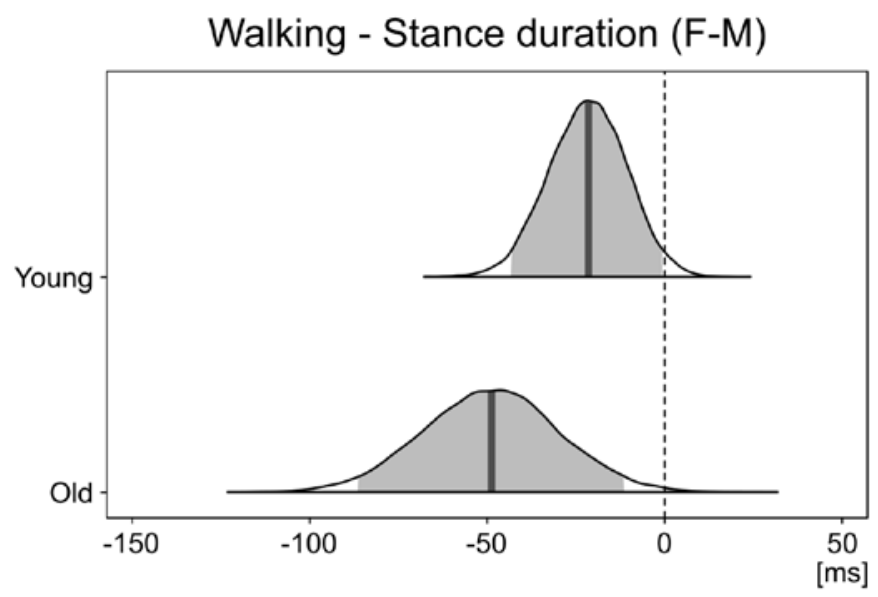

Figure S1. Post-hoc 95\% credible intervals for the estimated differences in stance duration between females (F) and males (M). The 95\% credible intervals and their probability 810 distributions (shaded areas) describe the post-hoc analysis of the interaction between sex and age on the stance duration. Negative values indicate shorter stance times in females than in males. 


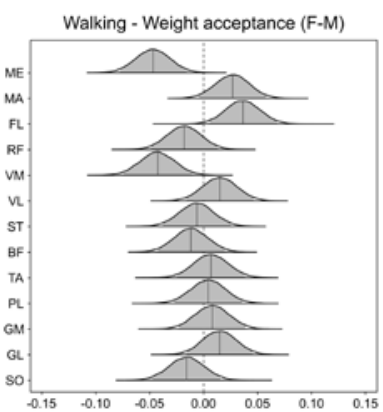

Running - Weight acceptance (F-M)

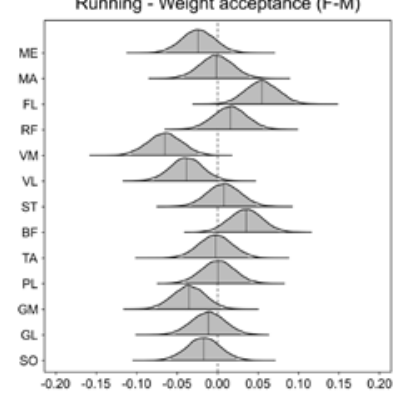

Walking - Propulsion (F-M)

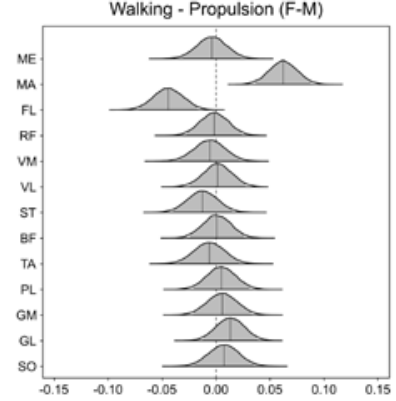

Running - Propulsion (F-M)

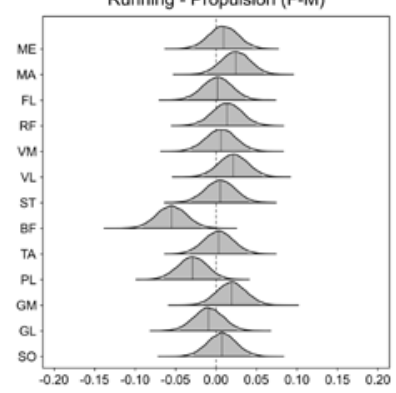

Walking - Early swing (F-M)

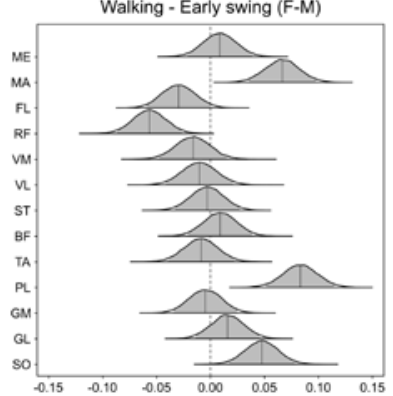

Running - Early swing (F-M)

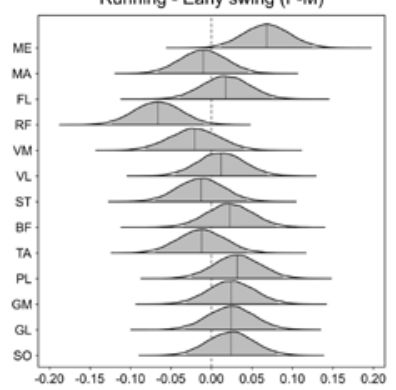

Walking - Late swing (F-M)

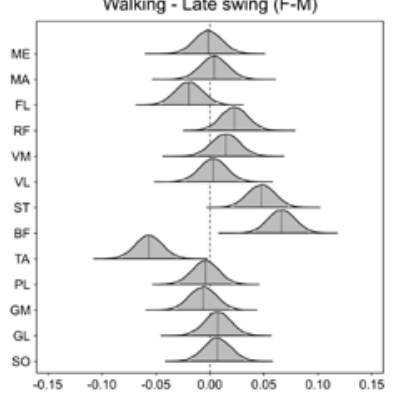

Running - Late swing (F-M)

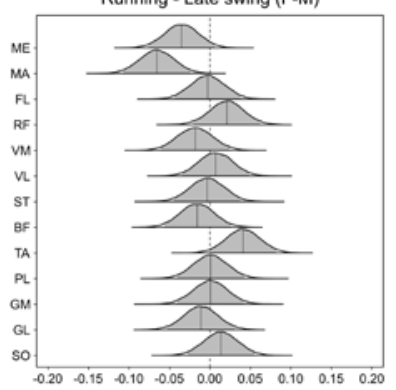

Figure S2. Post-hoc 95\% credible intervals for the estimated differences in motor modules

815 between females (F) and males (M). The 95\% credible intervals and their probability distributions (shaded areas) describe the post-hoc analysis of the interaction between sex and muscle on the motor modules. Positive values indicate higher muscle contribution in females than in males. Muscle abbreviations: $\mathrm{ME}=$ gluteus medius, $\mathrm{MA}=$ gluteus maximus, $\mathrm{FL}=$ tensor fascice late, $\mathrm{RF}=$ rectus femoris, VM=vastus medialis, $\mathrm{VL}=$ vastus lateralis, $\mathrm{ST}=$ semitendinosus, $820 \mathrm{BF}=$ biceps femoris, $\mathrm{TA}=$ tibialis anterior, $\mathrm{PL}=$ peroneus longus, $\mathrm{GM}=$ gastrocnemius medialis, $\mathrm{GL}=$ gastrocnemius lateralis, $\mathrm{SO}=$ soleus . 

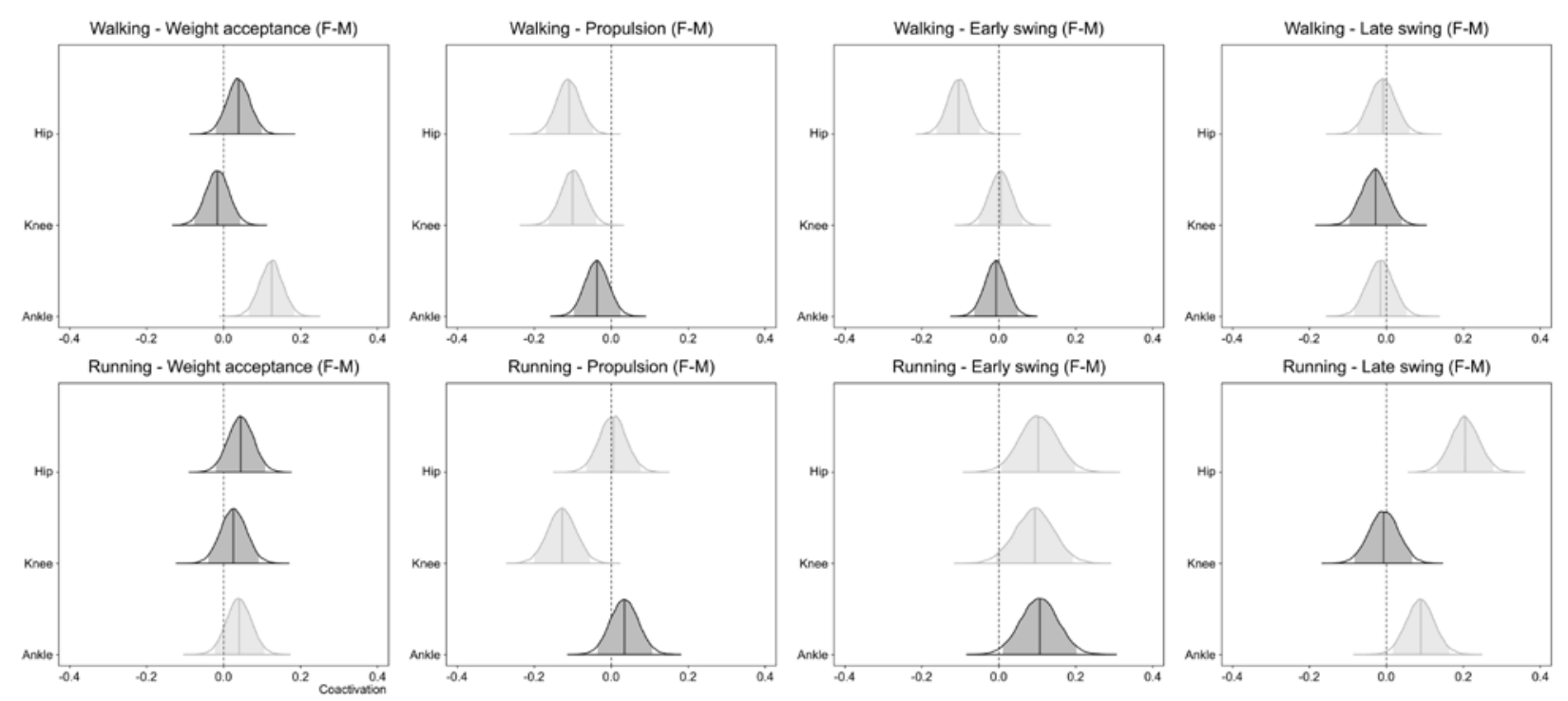

Figure S3. Post-hoc 95\% credible intervals for the estimated differences in coactivation

between females (F) and males (M). The 95\% credible intervals and their probability distributions (shaded areas) describe the post-hoc analysis of the interaction between sex and joint on the coactivation index. Joints crossed by the most important muscles contributing to each muscle synergy are highlighted. Negative values indicate lower coactivation index in females than in males (please note that coactivation index values close to zero mean that muscle activity comes exclusively from extensors; values close to one mean that activity comes only from flexors; values of 0.5 indicate perfect coactivation of flexors and extensors, meaning that the average module values of flexors for that joint are the same as the extensors'). 


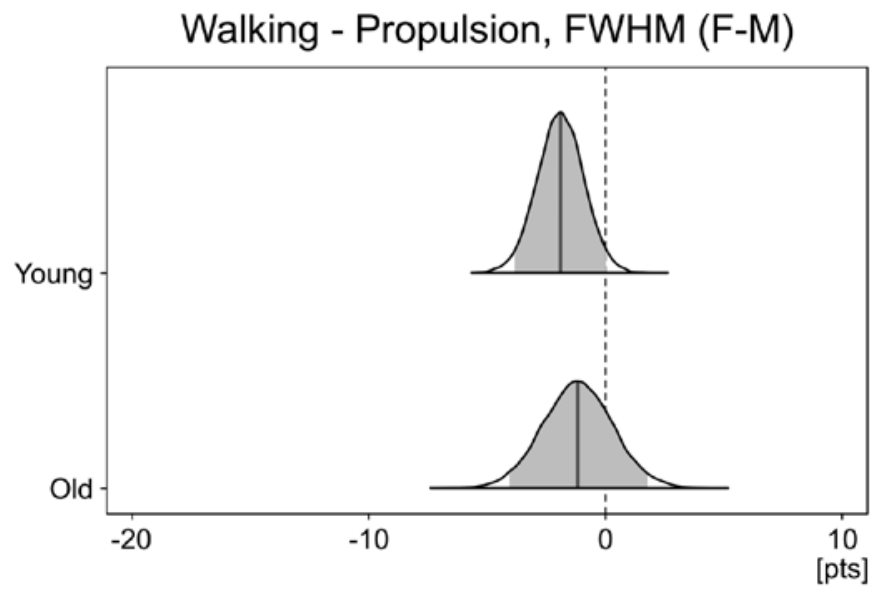

Figure S4. Post-hoc $95 \%$ credible intervals for the estimated differences in full width at half maximum between females (F) and males (M). The 95\% credible intervals and their probability distributions (shaded areas) describe the post-hoc analysis of the interaction between sex and age on the full width at half maximum (FWHM). Negative values indicate narrower primitives in 840 females than in males. 
A Walking - Higuchi's fractal dimension (F-M)
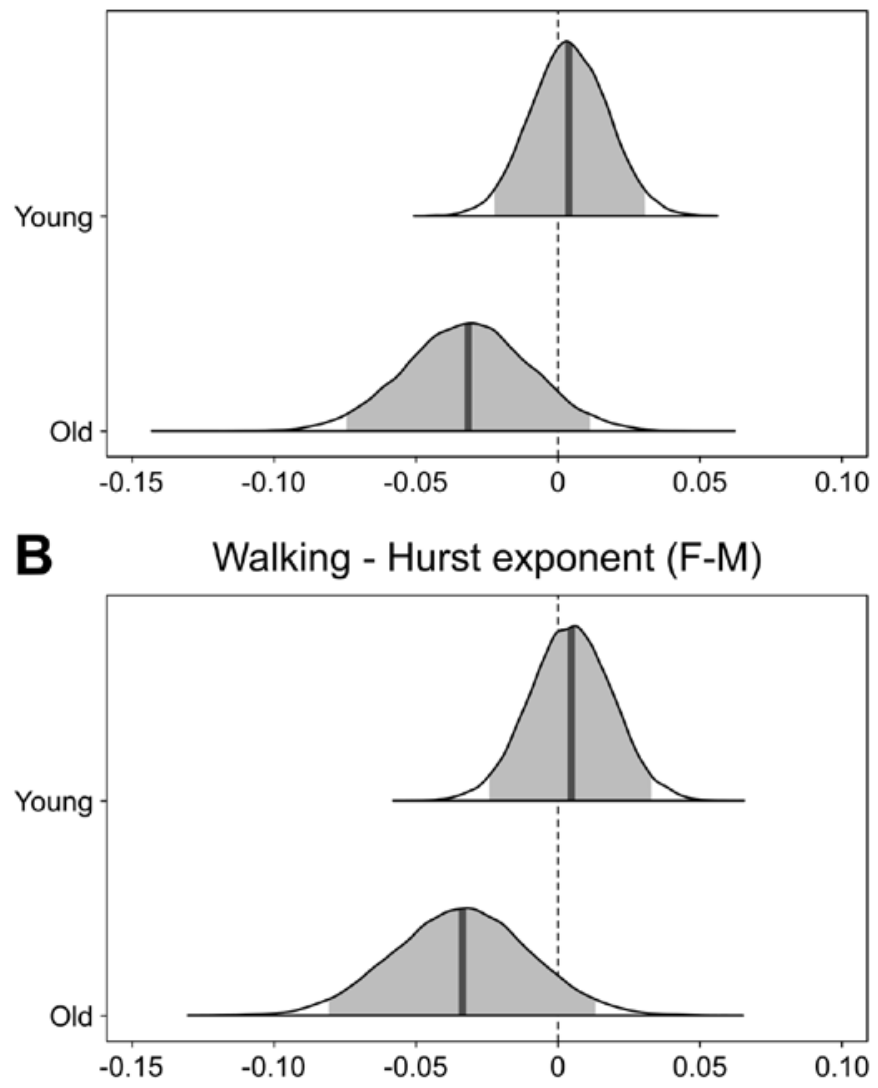

Figure S5. Post-hoc 95\% credible intervals for the estimated differences in Higuchi's fractal dimension and Hurst exponent between females (F) and males (M). (A) The 95\% credible intervals and their probability distributions (shaded areas) describe the post-hoc analysis of the interaction between sex and age on the Higuchi’s fractal dimension. Positive values indicate higher fractal dimension in females than in males. (B) The same as in panel (A), but for the Hurst exponent. 
Walking
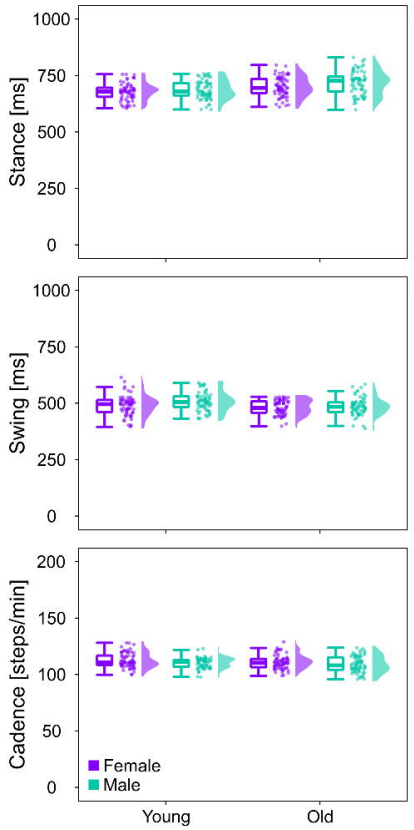

Running
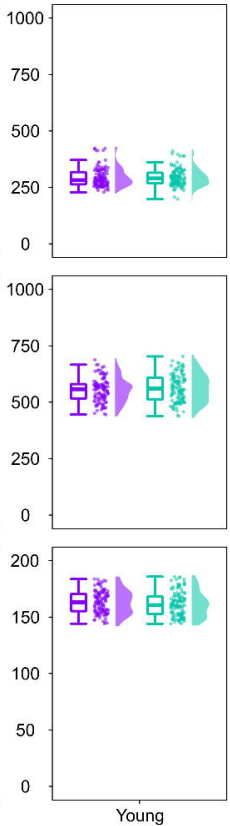


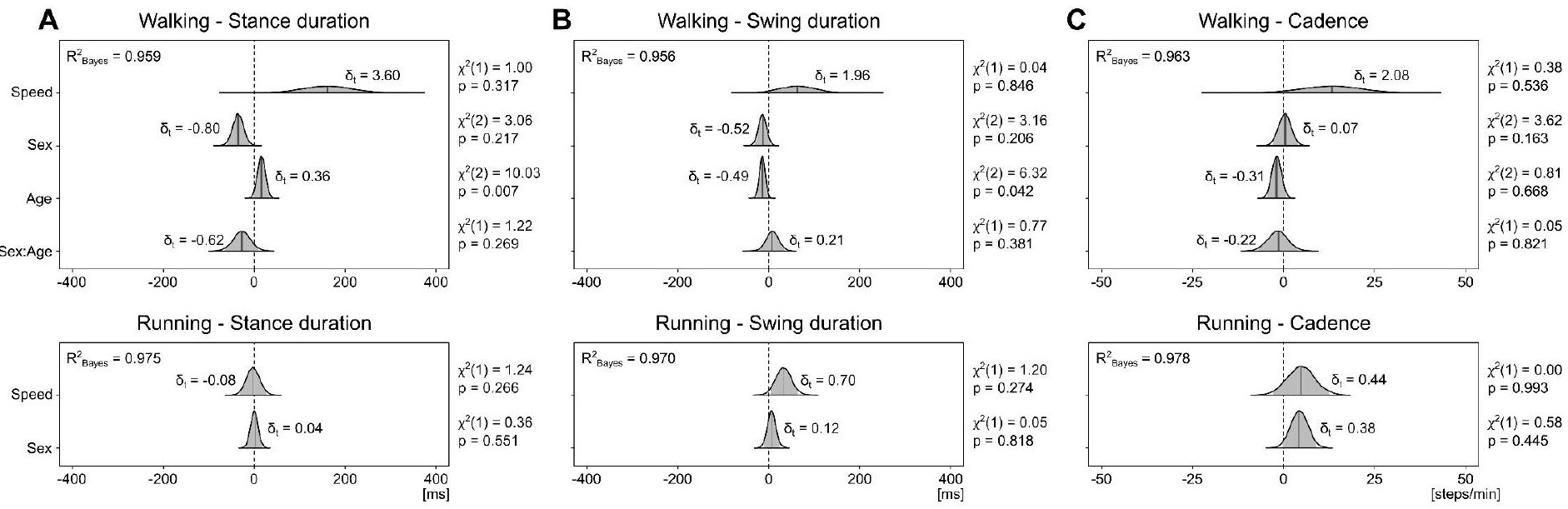




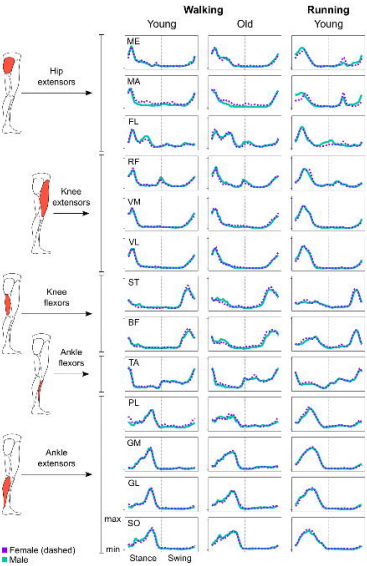


Walking, young females

Motor modules

Motor primitives

Weight acceptance
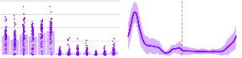

Propulsion
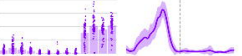

Early swing
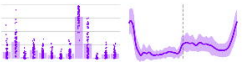
mex

Late

swing

militi ins.

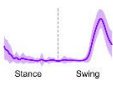

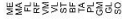

Walking, young males

Motor modules

Motor primitives

Weight acceptance
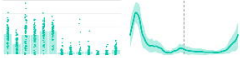

Propulsion

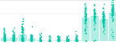

Early

swing
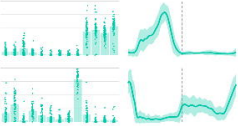

Late swing $\max$
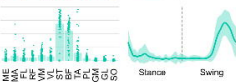

Walking, old females

Motor modules

$1+11$

ifilifitiol

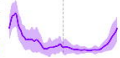

ilitibilil
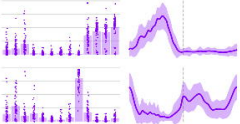

$\max$
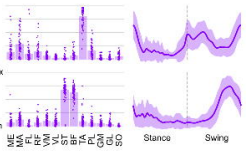

Walking, old males

Motor modules

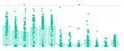

Motor pnmitives
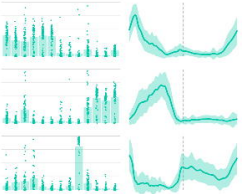

$\max$

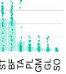

molitilitión.

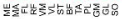

Running, young females

Motor modules

Motor primitives

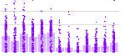

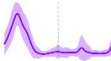

inilibiling

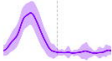

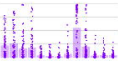

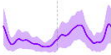

$\max$

miltibilitis

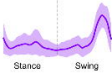

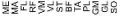

Running, young males

Motor modules

Motor primitives

(I)

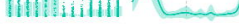

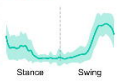
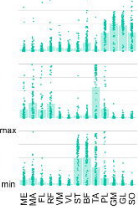
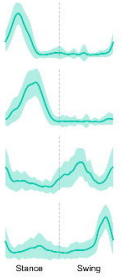


\section{A}

Weight acceptance Motor modules Motor primitives I

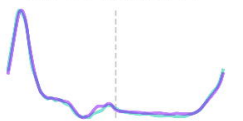

Propulsion

-
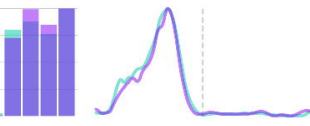

Early

swing
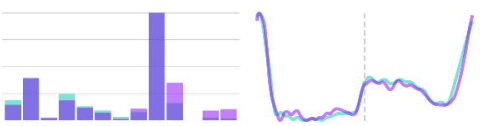

Late

swing

$$
\begin{aligned}
& \min =-1=0
\end{aligned}
$$

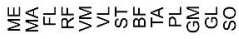

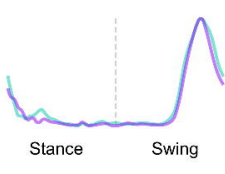

Walking, old participants Motor modules

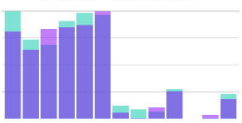

Motor primitives
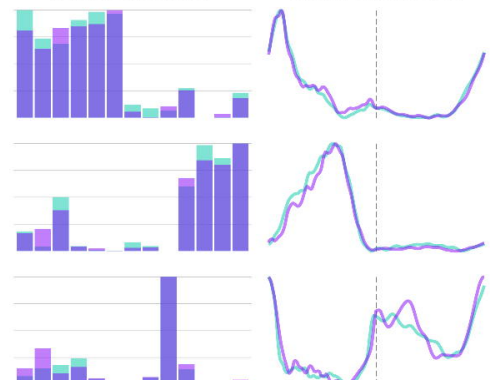

$\max$

$\min$

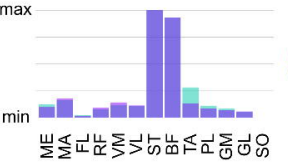

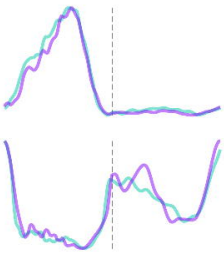

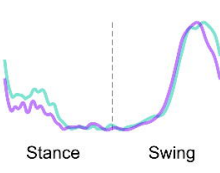

Running, young participants Motor modules

Motor primitives

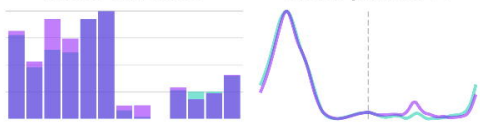

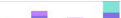

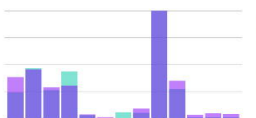

$\max$
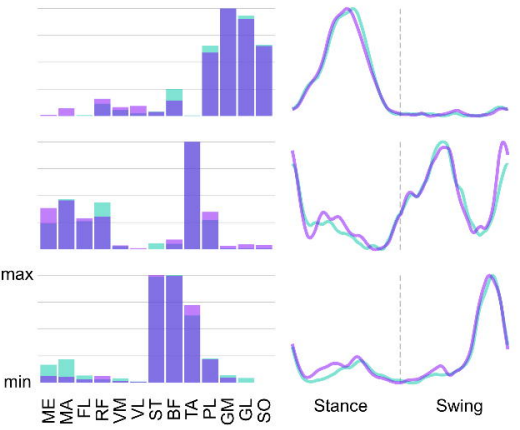

B

- Female

Male
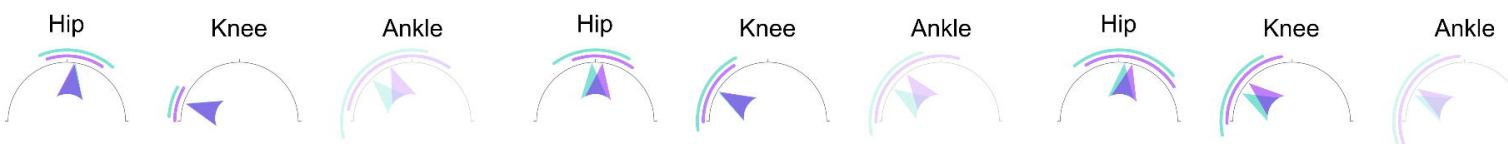

Propulsion
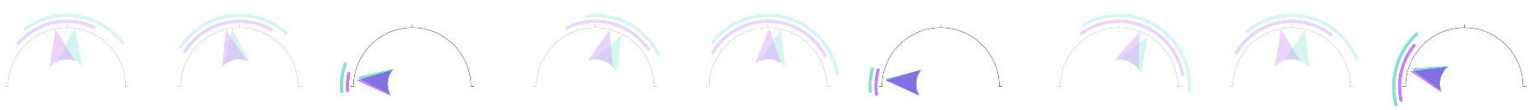

Early

swing
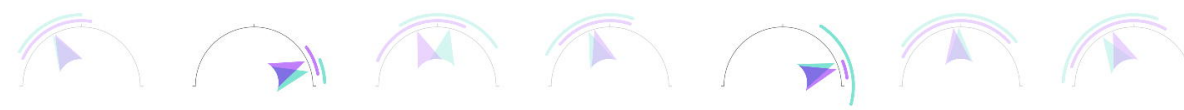

Late

swing

$$
\text { Coactivation }
$$
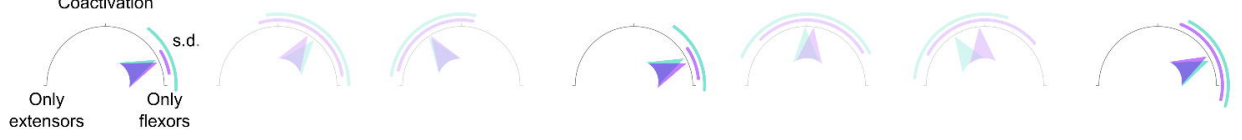
A
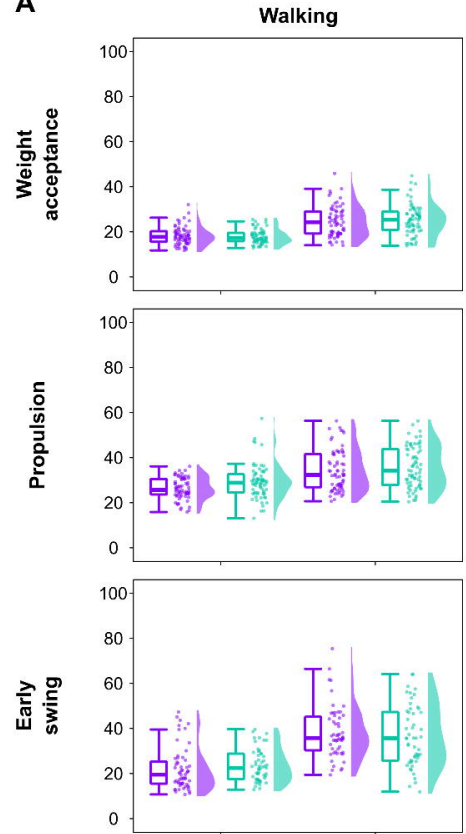

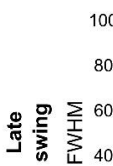

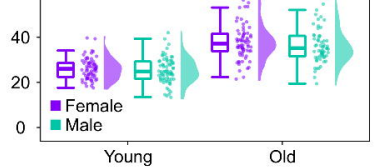

B
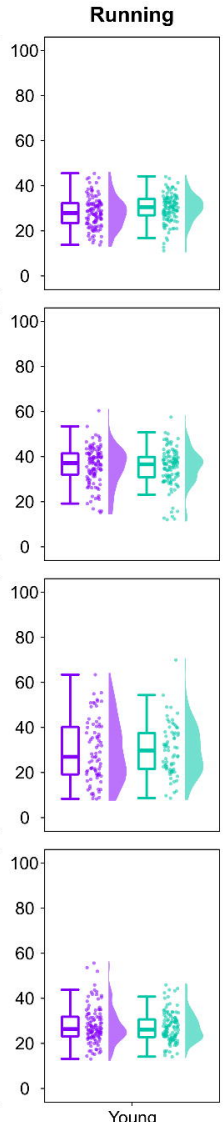

Walking
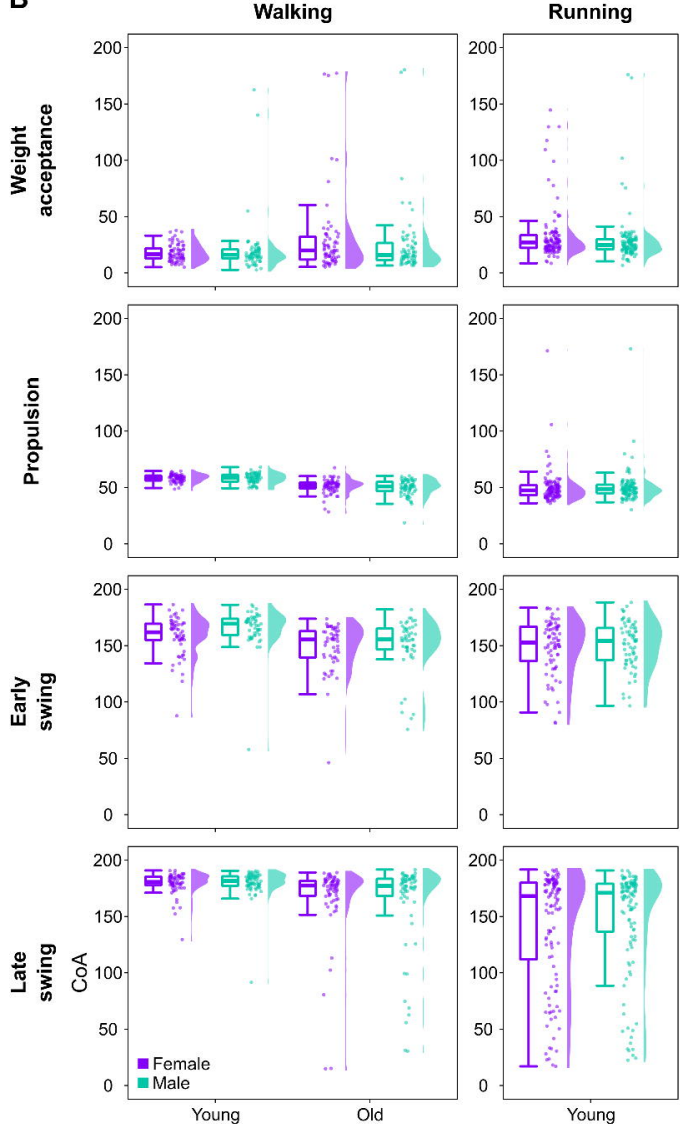
A

Walking, young

Walking, old

Running, young

Weight

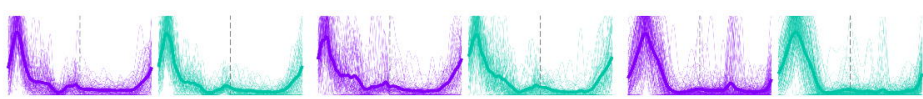

Propulsion

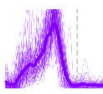

$\Lambda$

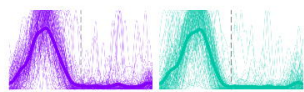

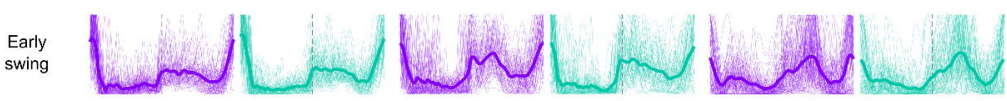

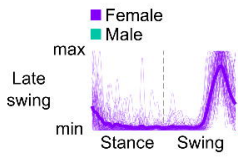

\section{B}
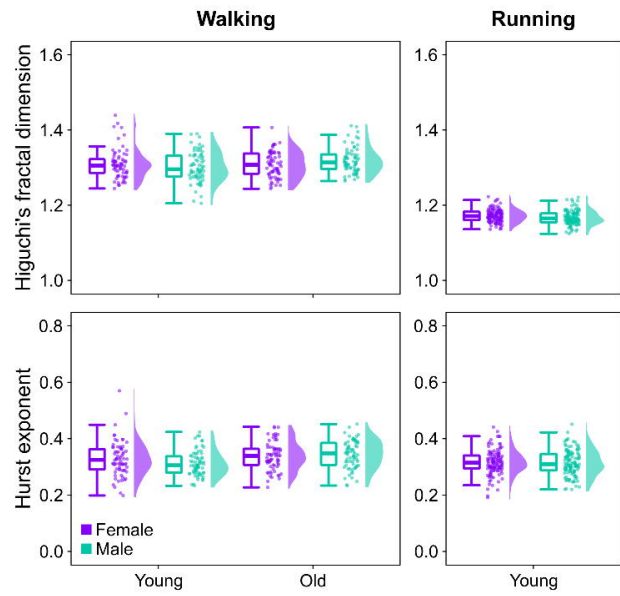
A Walking - Higuchi's fractal dimension

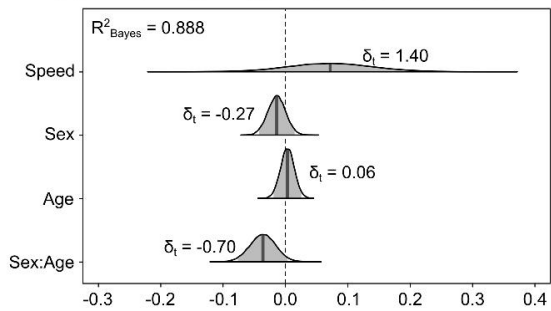

\section{Running - Higuchi's fractal dimension}

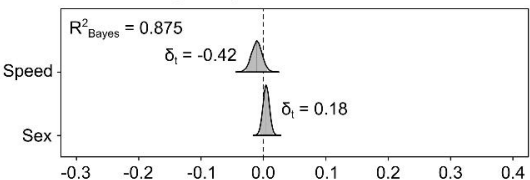

$\chi^{2}(1)=0.38$

$\mathrm{p}=0.537$

$\chi^{2}(2)=2.82$

$\mathrm{p}=0.244$

$\chi^{2}(2)=4.60$

$p=0.100$

$\chi^{2}(1)=0.38$

$p=0.537$
B

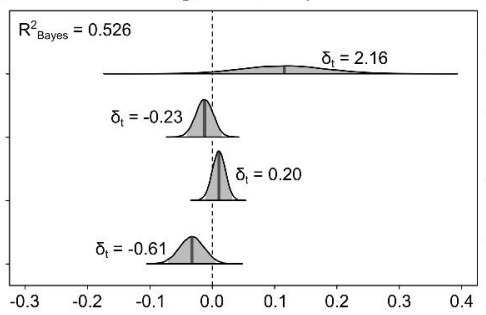

Running - Hurst exponent

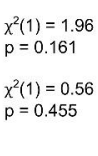

Walking - Hurst exponent

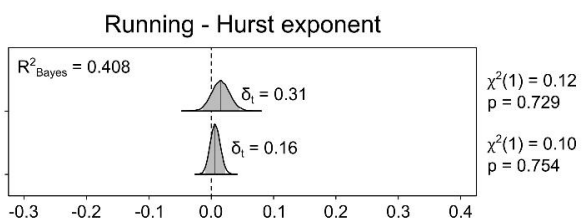




\section{Walking - Stance duration (F-M)}

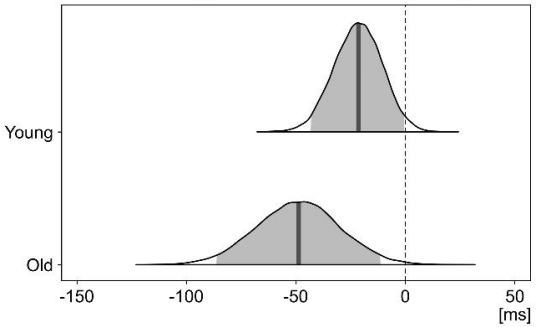


Walking - Weight acceptance (F-M)

Walking - Propulsion (F-M)

Walking - Early swing (F-M)

Hip

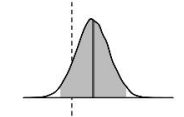

Knee

Ankle

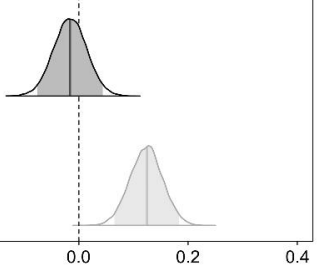

Running - Weight acceptance (F-M)

Hip

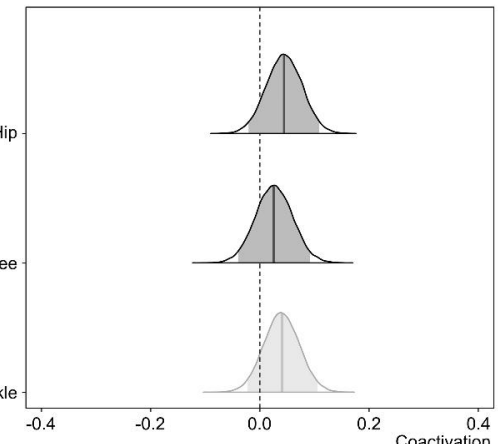

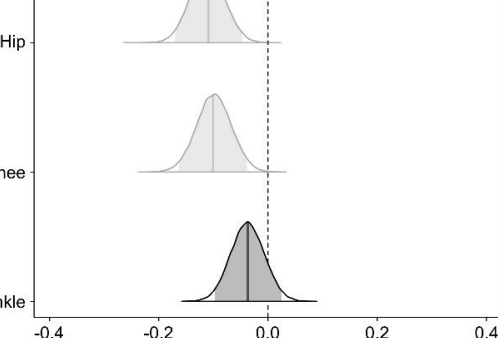

Running - Propulsion (F-M)

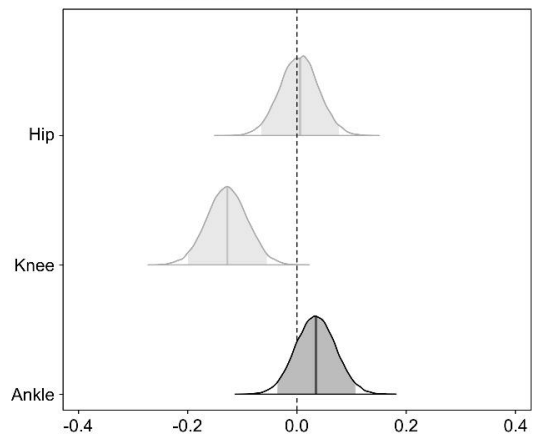

Running - Early swing (F-M)

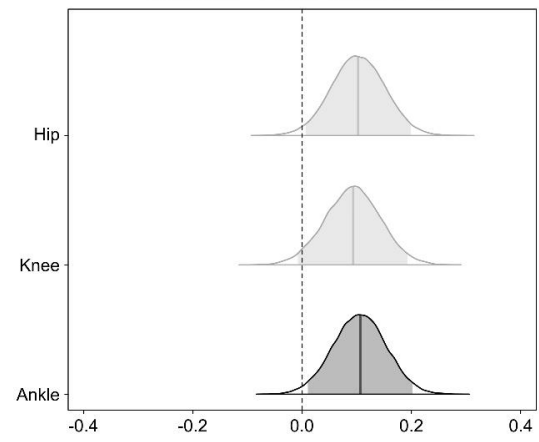

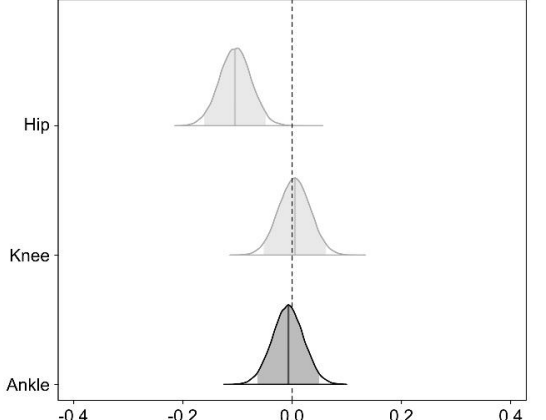

Ankle

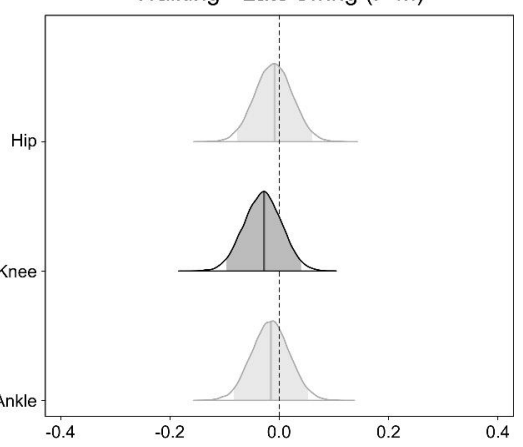

Running - Late swing (F-M)

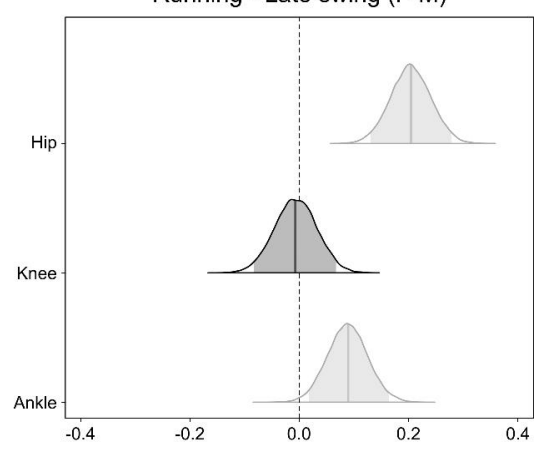




\section{Walking - Propulsion, FWHM (F-M)}

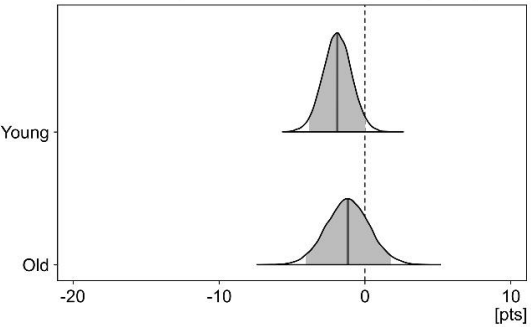


A Walking - Higuchi's fractal dimension (F-M)

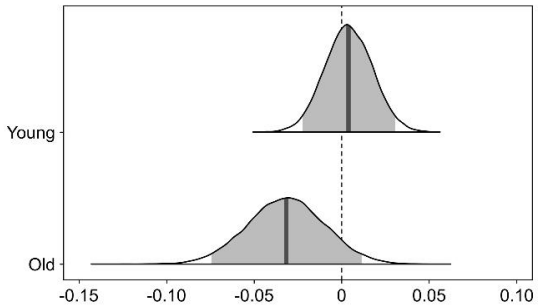

\section{Walking - Hurst exponent (F-M)}

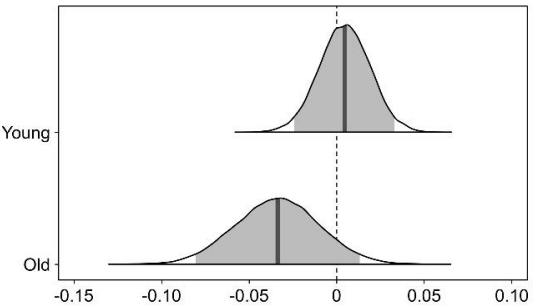

